\title{
Determinants of Non-Adherence to the Medications for Dyslipidemia: A Systematic Review
}

\author{
João Lopes (D) \\ Paulo Santos (iD) ${ }^{1,2}$ \\ 'Department of Community Medicine, \\ Information and Health Decision Sciences \\ (MEDCIDS), Faculty of Medicine, \\ University of Porto, Porto, Portugal; \\ ${ }^{2}$ CINTESIS - Center for Health \\ Technology and Services Research, \\ University of Porto, Porto, Portugal
}

Purpose: Dyslipidemia is a major cardiovascular risk factor, and its control leads to less cardiovascular events. Many patients will need some medications to achieve ideal targets. Non-adherence to medications is a complex problem with high impact on their effectiveness. This study aims to identify the determinants of non-adherence to medications in patients with dyslipidemia.

Patients and Methods: We conducted a systematic review. PubMed and Scopus databases were searched for original articles, published between 2000 and 2020, using the MeSH terms "Dyslipidemias" and "Medication Adherence".

Results: From the initial 3502 identified articles, we selected 46 to include in the final qualitative synthesis. The determinants associated with non-adherence were lower age $(\leq 50$ years), female sex, African American ethnicity, smoking habits, being a new user of lipidlowering medications, reporting or having concerns about lipid-lowering medication side effects and some comorbidities (chronic obstructive pulmonary disease, Alzheimer's disease/ dementia, depression and diabetes). On the contrary, adherence is higher in older patients, alcohol drinking habits, taking $\beta$-blockers, having a higher number of comorbidities, having a history of cardiovascular events, cardiac interventions or revascularization procedures, having health insurance and having more provider follow-up visits.

Conclusion: There are important identifiable determinants of non-adherence in patients with dyslipidemia. These patients benefit from a specific approach to minimize the problem and maximize the potential benefit of the prescription.

Keywords: medication adherence, dyslipidemias, causality, epidemiologic factors, heart disease risk factors

\section{Introduction}

Dyslipidemia is an important risk factor for developing cardiovascular diseases (CVD), the leading cause of death in the world, both for ischemic heart disease ${ }^{1}$ and stroke. ${ }^{1}$ In Europe, CVD account for more than 4 million deaths per year, about $45 \%$ of the total. ${ }^{2}$ In this study, we consider dyslipidemia as a broader term, covering a wide range of lipid abnormalities, and not merely having elevated total cholesterol and low-density lipoprotein. ${ }^{3}$ Therefore, patients were considered as having dyslipidemia if they had any lipid abnormalities that justified treatment, including secondary prevention of cardiovascular events and laboratory confirmation of hypercholesterolemia, hypertriglyceridemia or both. Medication non-adherence is a serious and complex problem. Adherence is
Correspondence: João Lopes

Department of Community Medicine, Information and Health Decision Sciences (MEDCIDS), Faculty of Medicine,

University of Porto, Alameda Prof. Hernâni Monteiro, Porto, 4200-319, Portugal

Tel +35I 220426600

Email joaomoraislopes@gmail.com 
defined as the extent to which the patients' behavior correspond to the agreed recommendation of their doctors, both in taking the medications, following a diet or executing lifestyle changes. ${ }^{4}$ It depends on several variables related to the condition itself, the therapeutics, the factors related to the patient, including social and cultural features and the health system-related factors. It develops in an interactive triangle of patient, healthcare provider and healthcare system, where each one insert both barriers and facilitators, complementary and interdependently for the outcome. ${ }^{5}$

About half of patients do not take the medications accordingly to the doctor's prescription, ${ }^{6}$ with a significant impact on treatment's efficacy. Also, nonadherence presents an important economic impact, with annual costs varying from $3347 \$$ to $19,472 \$$ per patient. $^{7}$

In the context of CVD, non-adherence to medications is associated with significant increase in CVD events ${ }^{8}$ and higher economic costs. ${ }^{9}$ It may justify about $9 \%$ of all CVD events. ${ }^{10}$

It is crucial to take action to improve adherence to medications, namely in patients with dyslipidemia. The risk of non-adherence varies with several factors including patients' characteristics, the disease itself and constraints of health system organizations. ${ }^{5,11}$ The capacity to predict this risk is an important help to allow a better adjustment of the personalized approach in the decision-making of the clinical consultation. Patients with a predictably higher risk of non-adherence benefit from better attention and greater intervention to improve it, thus better controlling dyslipidemia and consequently the cardiovascular risk. Therefore, it is essential to perceive and better characterize this group of patients with higher risk of non-adherence.

The aim of our study is to identify the determinants of non-adherence to medications in patients with dyslipidemia.

\section{Patients and Methods}

In January 2021, we performed a literature search in the PubMed and Scopus databases for all the records published from January 2000 to December 2020 (including), in English, Portuguese and Spanish, using the MeSH terms "Dyslipidemias" and "Medication Adherence". To organize this systematic review, we used the Preferred Reporting Items for Systematic Reviews and MetaAnalysis (PRISMA). ${ }^{12}$ All the articles and references were managed using the citation manager software EndNote (version 20).

\section{Study Selection and Data Extraction}

The process for selecting studies involved two phases: screening by title and abstract; and assessing the eligibility in full text. Data collected from each study and selected for the final qualitative synthesis, were the study identification (author and citation), study design and setting, patient population size for each study, the type of measure of adherence and the determinants associated with nonadherence. Article screening and data collection were conducted by two independent authors. All differences were discussed and decided by mutual agreement.

\section{Inclusion Criteria}

We included studies on patients with dyslipidemia that evaluated the adherence to any dyslipidemia medication, including statins, or the predictors of adherence to medications. We did not include studies evaluating adherence to dyslipidemia guidelines, interventions to improve adherence or validation of such interventions, studies for evaluation or validation of methods to measure medication adherence, studies on adherence trends, and studies of cost or outcome analysis.

\section{Exclusion Criteria}

We excluded reviews and case reports, studies involving pediatric populations, studies that assessed concomitantly the adherence to medications to other cardiovascular risk factors beyond dyslipidemia (including antidiabetics and antihypertensives), and studies that limited the population to specific groups, like sex or diseases beyond CVD or cardiovascular risk factors beyond dyslipidemia.

\section{Assessment of Study Quality}

To assess the quality of the studies, we used an adapted version of the 2018 Quality Assessment Tool for Observational Cohort and Cross-Sectional Studies from the National Heart Lung and Blood Institute. ${ }^{13}$ We choose 4 questions out of 14 that compose the tool, based on the adequacy for the proposal of this study: "1. Was the research question or objective in this paper clearly stated?", "2. Was the study population clearly specified and defined?", "11. Were the outcome measures (dependent variables) clearly defined, valid, reliable, and implemented consistently across all study participants?" and " 14 . Were key potential confounding variables measured and adjusted statistically for their impact on the relationship between exposure(s) and outcome(s)?". We classified each question in "Yes" (1) or "No" (0) for each study. The resulting score ranged from 0 to 4 . Only studies with 2 or more points were 
considered with the sufficient quality and therefore accepted for inclusion in the final analysis.

\section{Ethical Concerns}

Previous ethical appraisal by the ethics committee was not mandatory due to the review nature of this research. Nevertheless, we assumed the commitment to the ethical principles of the Helsinki Declaration, in the conception, analysis and discussion of our results.

\section{Results}

We identified 1954 records from PubMed search database and 1548 from the Scopus search database, in a total sample of 2644 articles, after eliminating the duplicates. The screening by title and abstract excluded a total of 2507 records, and the remaining 137 records were selected for a full-text assessment. From this final assessment, 90 articles were excluded. In this phase, 47 studies remained for the inclusion on the qualitative synthesis. The process is summarized in Figure 1.

\section{Quality Assessment}

The results of the quality assessment of each article are described in Table 1. From the 47 studies analyzed: 29 had a score of 4,16 had a score of 3,1 had a score of 2 and 1 had a score of 1 . Consequently, only one study was excluded, and the remaining 46 were included in the final qualitative synthesis.

Two authors read independently all 46 studies, searching for outcomes of non-adherence. The studies evaluated non-adherence, adherence, discontinuation, or persistence with medication. All articles were interpreted in a case-bycase approach and considered in terms of non-adherence. The outcomes of non-adherence searched preferentially were statistical measures: odds ratio, risk ratio and hazard ratio. Many studies only presented a qualitative assessment of the results that were also considered.

All the variables assessed for each of the 46 articles selected for the final qualitative synthesis are summarized in Table 2. From these 46 observational studies analyzed, 25

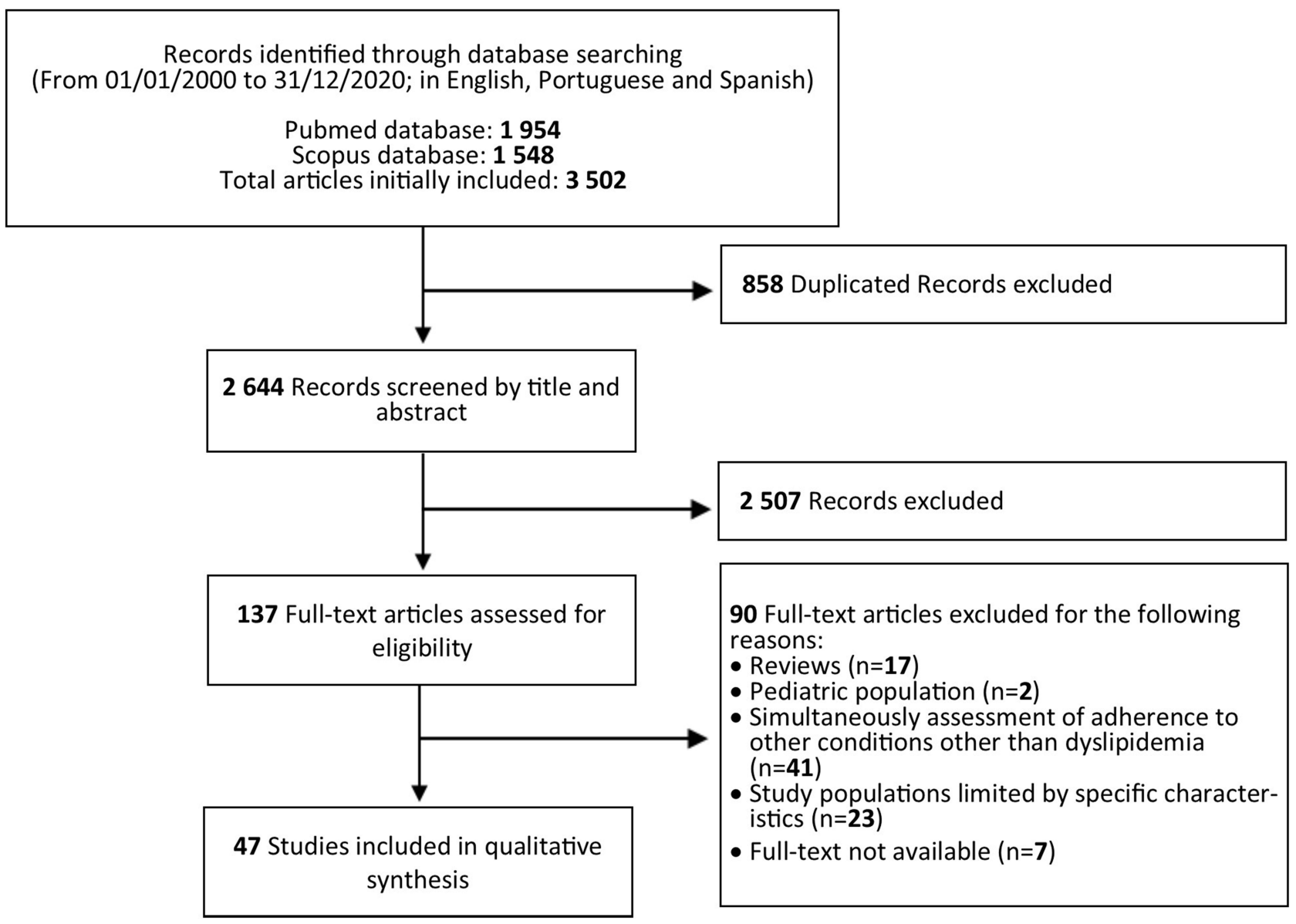

Figure I PRISMA flow diagram for the review.

Notes: PRISMA figure adapted from Liberati A, Altman D, Tetzlaff J, et al. The PRISMA statement for reporting systematic reviews and meta-analyses of studies that evaluate health care interventions: explanation and elaboration. Journal of Clinical Epidemiology. 2009;62(I0). Creative Commons. ${ }^{12 .}$ 
Table I Quality Assessment of the Articles

\begin{tabular}{|c|c|c|c|c|}
\hline Article Identification - Author (Year) & Question I & Question 2 & Question II & Question 14 \\
\hline Kiortsis et al $(2000)^{14}$ & Yes & Yes & Yes & No \\
\hline Mansur et al $(2001)^{15}$ & Yes & Yes & Yes & No \\
\hline O'Donnell et al $(2001)^{16}$ & Yes & Yes & Yes & No \\
\hline Yang et al $(2003)^{17}$ & Yes & No & Yes & Yes \\
\hline Stilley et al $(2004)^{18}$ & Yes & Yes & Yes & No \\
\hline Benner et al $(2005)^{19}$ & Yes & Yes & Yes & Yes \\
\hline Caspard et al $(2005)^{20}$ & Yes & Yes & Yes & Yes \\
\hline Di Martino et al $(2005)^{21}$ & Yes & Yes & Yes & Yes \\
\hline Huser et al $(2005)^{22}$ & Yes & No & No & No \\
\hline Batal et al $(2007)^{23}$ & Yes & Yes & Yes & Yes \\
\hline McGinnis et al $(2007)^{24}$ & Yes & Yes & Yes & No \\
\hline Natarajan et al $(2007)^{25}$ & Yes & Yes & Yes & No \\
\hline Ye et al $(2007)^{26}$ & Yes & Yes & Yes & Yes \\
\hline Chodick et al $(2008)^{27}$ & Yes & Yes & Yes & Yes \\
\hline Vinker et al $(2008)^{28}$ & Yes & Yes & Yes & Yes \\
\hline Latry et al $(201 \mathrm{I})^{29}$ & Yes & Yes & Yes & No \\
\hline Wong et al $(20 \mathrm{II})^{30}$ & Yes & Yes & Yes & Yes \\
\hline Wong et al $(20 \mathrm{II})^{3 !}$ & Yes & Yes & Yes & Yes \\
\hline Brogaard et al $(2012)^{32}$ & Yes & Yes & Yes & No \\
\hline Cheetham et al $(2013)^{33}$ & Yes & Yes & Yes & Yes \\
\hline Christian et al $(2013)^{34}$ & Yes & Yes & Yes & Yes \\
\hline Xie et al $(2013)^{35}$ & Yes & Yes & Yes & Yes \\
\hline Cicero et al $(2014)^{36}$ & Yes & Yes & Yes & Yes \\
\hline Halava et al $(2014)^{37}$ & Yes & Yes & Yes & Yes \\
\hline Gaisenok et al $(2015)^{38}$ & Yes & Yes & No & No \\
\hline Halava et al $(2015)^{39}$ & Yes & Yes & Yes & Yes \\
\hline Korhonen et al $(2015)^{40}$ & Yes & Yes & Yes & Yes \\
\hline Warren et al $(2015)^{4 l}$ & Yes & Yes & Yes & Yes \\
\hline Korhonen et al $(2016)^{42}$ & Yes & Yes & Yes & Yes \\
\hline Kronish et al $(2016)^{43}$ & Yes & Yes & Yes & Yes \\
\hline Tokgözoğlu et al $(2016)^{44}$ & Yes & Yes & Yes & No \\
\hline Al-Foraih et al $(2017)^{45}$ & Yes & Yes & Yes & Yes \\
\hline Booth et al $(2017)^{46}$ & Yes & Yes & Yes & No \\
\hline Devaraj et al $(2017)^{47}$ & Yes & Yes & Yes & Yes \\
\hline Hickson et al $(2017)^{48}$ & Yes & Yes & Yes & Yes \\
\hline Wawruch et al $(2017)^{49}$ & Yes & Yes & Yes & No \\
\hline Chung et al $(2018)^{50}$ & Yes & Yes & Yes & Yes \\
\hline do Nascimento et al $(20 \mid 8)^{51}$ & Yes & Yes & Yes & Yes \\
\hline Haddad et al $(2018)^{52}$ & Yes & Yes & Yes & Yes \\
\hline Kriegbaum et al $(2018)^{53}$ & Yes & Yes & Yes & Yes \\
\hline Ofori-Asenso et al $(2018)^{54}$ & Yes & Yes & Yes & No \\
\hline Chen et al $(2019)^{55}$ & Yes & Yes & Yes & Yes \\
\hline Phan et al $(2019)^{56}$ & Yes & Yes & Yes & Yes \\
\hline Waßmuth et al $(2019)^{57}$ & Yes & Yes & Yes & No \\
\hline Bruckert et al $(2020)^{58}$ & Yes & Yes & Yes & No \\
\hline Seaman et al $(2020)^{59}$ & Yes & Yes & Yes & Yes \\
\hline Shakarneh et al $(2020)^{60}$ & Yes & Yes & Yes & No \\
\hline
\end{tabular}

Notes: Question I. "Was the research question or objective in this paper clearly stated?", Question 2. "Was the study population clearly specified and defined?", Question II. "Were the outcome measures (dependent variables) clearly defined, valid, reliable, and implemented consistently across all study participants?", Question I4. "Were key potential confounding variables measured and adjusted statistically for their impact on the relationship between exposure(s) and outcome(s)?". 
Table 2 Articles Assessed in Qualitative Synthesis

\begin{tabular}{|c|c|c|c|c|c|}
\hline Article & Study Design & Setting (Country) & $\begin{array}{l}\text { Patient } \\
\text { Population }\end{array}$ & Adherence Measure & $\begin{array}{c}\text { Determinants of Non- } \\
\text { Adherence }\end{array}$ \\
\hline $\begin{array}{l}\text { Kiortsis et al } \\
(2000)^{14}\end{array}$ & $\begin{array}{l}\text { Cross-sectional } \\
\text { study, by survey }\end{array}$ & $\begin{array}{l}\text { Clinic (Hôpital Pitié- } \\
\text { Salpétrière), Department of } \\
\text { Endocrinology and Metabolism, } \\
\text { Unit for Prevention of } \\
\text { Cardiovascular Diseases (Paris) }\end{array}$ & 193 patients & $\begin{array}{l}\text { Compliance was analyzed by } \\
\text { the percentage of pills missed } \\
\text { during the previous month. } \\
\text { Patients were divided into } \\
\text { three groups: (i) high } \\
\text { compliance (all prescribed pills } \\
\text { were taken), (ii) intermediate } \\
\text { group ( }<6 \% \text { of the prescribed } \\
\text { pills were missed) and (iii) low } \\
\text { compliance group (patients } \\
\text { who missed } \geq 6 \% \text { of the } \\
\text { prescribed pills) }\end{array}$ & $\begin{array}{l}\text { Increased non-adherence: } \\
\text { younger age, smoking habit, } \\
\text { perceived high frequency of } \\
\text { side effects, higher number of } \\
\text { prescription medications, bad } \\
\text { doctor-patient relationship, } \\
\text { perception that treatment is } \\
\text { not effective }\end{array}$ \\
\hline $\begin{array}{l}\text { Mansur et al } \\
(200 I)^{15}\end{array}$ & $\begin{array}{l}\text { Prospective } \\
\text { observational study }\end{array}$ & $\begin{array}{l}\text { Coronariopathy Unit at the } \\
\text { Heart Institute, Medical School, } \\
\text { University of São Paulo (InCor) } \\
\text { (Brazil) }\end{array}$ & 207 patients & $\begin{array}{l}\text { Nonadherence was classified } \\
\text { as I) lack of understanding by } \\
\text { patients of the physician's } \\
\text { instructions for taking the } \\
\text { medication, 2) due to side } \\
\text { effects, 3) non-utilization of the } \\
\text { medication for other reasons }\end{array}$ & $\begin{array}{l}\text { Increased non-adherence: } \\
\text { high cost of the medication, } \\
\text { lack of proper instruction on } \\
\text { how to use medication }\end{array}$ \\
\hline $\begin{array}{l}\text { O'Donnell } \\
\text { et al } \\
(2001)^{16}\end{array}$ & $\begin{array}{l}\text { Cross-sectional } \\
\text { study, by survey }\end{array}$ & $\begin{array}{l}\text { Multidisciplinary lipid clinic of } \\
\text { an integrated health care } \\
\text { system }\end{array}$ & 117 patients & $\begin{array}{l}\text { Adherence was determined by } \\
\text { the number of days (in } 30 \text { days) } \\
\text { that patients took their lipid- } \\
\text { lowering medications exactly } \\
\text { as prescribed. } 30 \text { days was } \\
\text { classified as adherent and } \leq 29 \\
\text { was classified as nonadherent }\end{array}$ & $\begin{array}{l}\text { Increased non-adherence: } \\
\text { perceived burden of the } \\
\text { disease } \\
\text { Decreased non-adherence: } \\
\text { older age, perceived benefits of } \\
\text { medication }\end{array}$ \\
\hline $\begin{array}{l}\text { Yang et al } \\
(2003)^{17}\end{array}$ & $\begin{array}{l}\text { Retrospective } \\
\text { cohort study }\end{array}$ & $\begin{array}{l}\text { UK residents enrolled with } \\
\text { selected general practitioners } \\
\text { (GPs) (UK) }\end{array}$ & $\begin{array}{l}22.408 \\
\text { patients }\end{array}$ & $\begin{array}{l}\text { Discontinuation of the original } \\
\text { LLD was identified when } \\
\text { a patient did not receive any } \\
\text { further LLD prescription } \\
\text { within } 3 \text { months after the } \\
\text { expected last date of any } \\
\text { previous LLD prescription }\end{array}$ & $\begin{array}{l}\text { Increased non-adherence: } \\
\text { female sex, smoking habit, less } \\
\text { follow-up visits, higher number } \\
\text { of non-cardiovascular } \\
\text { medications }(\geq 4) \\
\text { Decreased non-adherence: } \\
\text { older age, diabetes, taking } \\
\text { more classes of cardiovascular } \\
\text { medications }(\geq 3) \text {, statins or } \\
\text { fibrates as initial lipid-lowering } \\
\text { drug }\end{array}$ \\
\hline $\begin{array}{l}\text { Stilley et al } \\
(2004)^{18}\end{array}$ & Prospective study & $\begin{array}{l}\text { University of Pittsburgh } \\
\text { Medical Center (USA) }\end{array}$ & I58 patients & $\begin{array}{l}\text { Medication adherence data was } \\
\text { tracked with electronic cap } \\
\text { monitors, the Medication Event } \\
\text { Monitoring System (MEMS). } \\
\text { Percentage of days compliant } \\
\text { was used to measure } \\
\text { adherence in the primary } \\
\text { analyses. Adherence to dose } \\
\text { and schedule over the entire } \\
\text { period was also assessed. } \\
\text { Patients were considered } \\
\text { adherent if they had a rate of } \\
\text { compliance } \geq 80 \%\end{array}$ & $\begin{array}{l}\text { Increased non-adherence: } \\
\text { anxiety, depression } \\
\text { Decreased non-adherence: } \\
\text { higher conscientiousness, } \\
\text { higher estimated IQ, higher } \\
\text { visuospatial/constructional } \\
\text { ability }\end{array}$ \\
\hline
\end{tabular}


Table 2 (Continued).

\begin{tabular}{|c|c|c|c|c|c|}
\hline Article & Study Design & Setting (Country) & $\begin{array}{l}\text { Patient } \\
\text { Population }\end{array}$ & Adherence Measure & $\begin{array}{l}\text { Determinants of Non- } \\
\text { Adherence }\end{array}$ \\
\hline $\begin{array}{l}\text { Benner et al } \\
(2005)^{19}\end{array}$ & $\begin{array}{l}\text { Retrospective } \\
\text { cohort study }\end{array}$ & $\begin{array}{l}\text { Enrollees in a Southeastern } \\
\text { managed care plan (USA) }\end{array}$ & $\begin{array}{l}9.510 \\
\text { patients }\end{array}$ & $\begin{array}{l}\text { Proportion of days covered } \\
\text { (PDC). Subjects were } \\
\text { considered adherent if they } \\
\text { had a PDC of } \geq 80 \%\end{array}$ & $\begin{array}{l}\text { Increased non-adherence: } \\
\text { more months of therapy } \\
\text { duration, higher number of } \\
\text { prescription medications (9- } \\
43 \text { ) } \\
\text { Decreased non-adherence: } \\
\text { younger age, cardiovascular } \\
\text { intervention, less change in } \\
\text { LDL cholesterol concentration } \\
\text { at } 3 \text { months of therapy, } \\
\text { adherence } \geq 80 \% \text { during the } \\
\text { first } 3 \text { months of therapy }\end{array}$ \\
\hline $\begin{array}{l}\text { Caspard } \\
\text { et al } \\
(2005)^{20}\end{array}$ & $\begin{array}{l}\text { Retrospective } \\
\text { observational study }\end{array}$ & $\begin{array}{l}\text { Massachussets health } \\
\text { maintenance organization } \\
\text { (USA) }\end{array}$ & $\begin{array}{l}4.776 \\
\text { patients }\end{array}$ & $\begin{array}{l}\text { Adherence was measured by } \\
\text { Proportion of days covered } \\
\text { (PDC). Patients were } \\
\text { considered adherent if they } \\
\text { had a PDC of } \geq 80 \%\end{array}$ & $\begin{array}{l}\text { Increased non-adherence: } \\
\text { younger age ( }<50 \text { years), } \\
\text { female sex, baseline LDL } \\
\text { cholesterol } \geq 190 \mathrm{mg} / \mathrm{dL} \\
\text { Decreased non-adherence: } \\
\text { previous antihyperlipidemia } \\
\text { treatment }\end{array}$ \\
\hline $\begin{array}{l}\text { Di Martino } \\
\text { et al } \\
(2005)^{21}\end{array}$ & $\begin{array}{l}\text { Retrospective } \\
\text { observational study }\end{array}$ & $\begin{array}{l}\text { Patients attending general } \\
\text { practitioners in the Ravenna } \\
\text { area (Italy) }\end{array}$ & $\begin{array}{l}4.764 \\
\text { patients }\end{array}$ & $\begin{array}{l}\text { Poor adherence was defined as } \\
\text { a Standardized Mean Daily } \\
\text { Dose (SDD) }<0.8 \text { tablets/day } \\
\text { (less than } 80 \% \text { of the Defined } \\
\text { Daily Doses (DDD)) }\end{array}$ & $\begin{array}{l}\text { Increased non-adherence: } \\
\text { absence of previous } \\
\text { atherosclerotic disease, } \\
\text { absence of concomitant } \\
\text { diseases, smoking habit }\end{array}$ \\
\hline $\begin{array}{l}\text { Batal et al } \\
(2007)^{23}\end{array}$ & $\begin{array}{l}\text { Retrospective } \\
\text { cohort study }\end{array}$ & $\begin{array}{l}\text { Denver Health Medical Center } \\
\text { (USA) }\end{array}$ & $\begin{array}{l}3.386 \\
\text { patients }\end{array}$ & $\begin{array}{l}\text { Adherence was assessed by an } \\
\text { adherence score, calculated as } \\
\text { the number of days of drug } \\
\text { acquired divided by number of } \\
\text { days in the study. High } \\
\text { adherence was defined by an } \\
\text { adherence score of } \geq 80 \% \text { and } \\
\text { non-adherent as an adherence } \\
\text { score of }<80 \%\end{array}$ & $\begin{array}{l}\text { Increased non-adherence: } \\
\text { female sex, African American } \\
\text { ethnicity, Hispanic ethnicity } \\
\text { Decreased non-adherence: } \\
\text { age (per 10-year increase), } 60 \\
\text { versus } 30 \text { days supply } \\
\text { medication, each additional } \\
\text { comorbidity }\end{array}$ \\
\hline $\begin{array}{l}\text { McGinnis } \\
\text { et al } \\
(2007)^{24}\end{array}$ & $\begin{array}{l}\text { Phase I: } \\
\text { retrospective } \\
\text { medical record } \\
\text { review } \\
\text { Phase 2: cross- } \\
\text { sectional telephone } \\
\text { survey of patients } \\
\text { for whom a medical } \\
\text { record review was } \\
\text { completed }\end{array}$ & $\begin{array}{l}\text { Kaiser Permanente Colorado, } \\
\text { a health maintenance } \\
\text { organization that provides } \\
\text { integrated health care services } \\
\text { to members at } 18 \text { medical } \\
\text { offices in the Denver-Boulder } \\
\text { metropolitan area (USA) }\end{array}$ & $\begin{array}{l}\text { Phase I: } \\
435 \text { patients } \\
\text { Phase 2: } \\
242 \text { patients }\end{array}$ & $\begin{array}{l}\text { Proportion of days covered } \\
\text { (PDC). Cohorts were divided } \\
\text { into } 3 \text { PDC groups: } \geq 80 \%, 20 \text { - } \\
79 \% \text {, and }<20 \%\end{array}$ & $\begin{array}{l}\text { Increased non-adherence: } \\
\text { less provider follow-up visits, } \\
\text { less laboratory visits, bad } \\
\text { doctor-patient relationship, } \\
\text { reported adverse effects, being } \\
\text { unsure of the benefits of statins }\end{array}$ \\
\hline $\begin{array}{l}\text { Natarajan } \\
\text { et al } \\
(2007)^{25}\end{array}$ & $\begin{array}{l}\text { Cross-sectional } \\
\text { study, by survey }\end{array}$ & $\begin{array}{l}\text { Two academic family practice } \\
\text { clinics (Halifax, NS) }\end{array}$ & 284 patients & $\begin{array}{l}\text { Level of adherence to statin } \\
\text { medications was measured by } \\
\text { patients' self-report using the } \\
\text { 4-item Morisky scale, a 4-item } \\
\text { adherence measure }\end{array}$ & $\begin{array}{l}\text { Decreased non-adherence: } \\
\text { older age (>75 years), higher } \\
\text { number of other prescribed } \\
\text { medications (4-6), healthier } \\
\text { lifestyle (exercise and/or } \\
\text { healthy diet) }\end{array}$ \\
\hline
\end{tabular}

(Continued) 
Table 2 (Continued).

\begin{tabular}{|c|c|c|c|c|c|}
\hline Article & Study Design & Setting (Country) & $\begin{array}{c}\text { Patient } \\
\text { Population }\end{array}$ & Adherence Measure & $\begin{array}{l}\text { Determinants of Non- } \\
\text { Adherence }\end{array}$ \\
\hline $\begin{array}{l}\text { Ye et al } \\
(2007)^{26}\end{array}$ & $\begin{array}{l}\text { Longitudinal, } \\
\text { Retrospective, } \\
\text { Cohort Study }\end{array}$ & $\begin{array}{l}\text { Privately insured individuals } \\
\text { (USA) }\end{array}$ & $\begin{array}{l}5.548 \\
\text { patients }\end{array}$ & $\begin{array}{l}\text { Adherence was measured by } \\
\text { medication possession ratio } \\
\text { (MPR). Patients were } \\
\text { considered adherent if they } \\
\text { had an MPR of } \geq 80 \% \text {. }\end{array}$ & $\begin{array}{l}\text { Increased non-adherence: } \\
\text { copayment } \geq \$ 20 \text {, female sex, } \\
\text { depression, non-statin lipid } \\
\text { lowering drug use } \\
\text { Decreased non-adherence: } \\
\text { higher age, dyslipidemia }\end{array}$ \\
\hline $\begin{array}{l}\text { Chodick } \\
\text { et al } \\
(2008)^{27}\end{array}$ & $\begin{array}{l}\text { Retrospective } \\
\text { cohort study }\end{array}$ & $\begin{array}{l}\text { Maccabi Healthcare Services } \\
\text { (MHS) and from death } \\
\text { certificates (Israel) }\end{array}$ & $\begin{array}{l}229.918 \\
\text { patients }\end{array}$ & $\begin{array}{l}\text { Proportion of days covered } \\
\text { (PDC). Patients were } \\
\text { categorized into } 3 \text { groups: } \\
\text { adherent (PDC } \geq 80 \% \text {; partially } \\
\text { adherent (PDC } \geq 20 \% \text { and } \\
<80 \% \text { ), or nonadherent (PDC } \\
<20 \% \text { ) }\end{array}$ & $\begin{array}{l}\text { Increased non-adherence: } \\
\text { female sex, less time of } \\
\text { residence in Israel, chronic } \\
\text { obstructive pulmonary disease, } \\
\text { Alzheimer's disease, lower LDL } \\
\text { cholesterol (<130 mg/dL), } \\
\text { moderate statin therapeutic } \\
\text { efficacy, lowest socioeconomic } \\
\text { level } \\
\text { Decreased non-adherence: } \\
\text { age (per I0-year increase), } \\
\text { more health services utilization } \\
\text { (higher number of outpatient } \\
\text { visits and hospitalizations), } \\
\text { diabetes mellitus, hypertension, } \\
\text { taking ACEl, taking } \beta \text {-Blockers, } \\
\text { high statin therapeutic efficacy }\end{array}$ \\
\hline $\begin{array}{l}\text { Vinker et al } \\
(2008)^{28}\end{array}$ & $\begin{array}{l}\text { Retrospective } \\
\text { cohort study }\end{array}$ & $\begin{array}{l}\text { Central District of Clalit } \\
\text { Health } \\
\text { Services HMO (Israel) }\end{array}$ & $\begin{array}{l}47.680 \\
\text { patients }\end{array}$ & $\begin{array}{l}\text { Adherence was defined as } \\
\text { buying at least } 80 \% \text { of the } \\
\text { expected number of pills over } \\
\text { this a } 12 \text { month period. }\end{array}$ & $\begin{array}{l}\text { Increased non-adherence: } \\
\text { patients originary from } \\
\text { Ethiopia, new immigrants in } \\
\text { 1990 } \\
\text { Decreased non-adherence: } \\
\text { older age, female sex, chronic } \\
\text { diseases }\end{array}$ \\
\hline $\begin{array}{l}\text { Latry et al } \\
(2011)^{29}\end{array}$ & $\begin{array}{l}\text { Retrospective } \\
\text { cohort study }\end{array}$ & $\begin{array}{l}\text { French health insurance } \\
\text { system: the Caisse Nationale } \\
\text { d'Assurance Maladie des } \\
\text { Travailleurs Salariés (Cnam-TS) } \\
\text {, from Aquitaine region from } \\
\text { southwest France (France) }\end{array}$ & $\begin{array}{l}16.397 \\
\text { patients }\end{array}$ & $\begin{array}{l}\text { Adherence was assessed by } \\
\text { four criteria: (i) proportion of } \\
\text { days covered (PDC) by statins, } \\
\text { (ii) regularity of the treatment } \\
\text { over time, (iii) persistence of } \\
\text { treatment, and (iv) refill delay. } \\
\text { The PDC was estimated using } \\
\text { the "Continuous Multiple- } \\
\text { interval measures of } \\
\text { medication Availability" (CMA) } \\
\text { definition. A CMA < } 80 \% \text { was } \\
\text { considered as unsatisfactory }\end{array}$ & $\begin{array}{l}\text { Decreased non-adherence: } \\
\text { associated cardiovascular risk } \\
\text { factors }\end{array}$ \\
\hline $\begin{array}{l}\text { Wong et al } \\
(2011)^{30}\end{array}$ & $\begin{array}{l}\text { Retrospective } \\
\text { cohort study }\end{array}$ & $\begin{array}{l}\text { Public, primary care clinics } \\
\text { (Hong Kong, China) }\end{array}$ & $\begin{array}{l}11.042 \\
\text { patients }\end{array}$ & $\begin{array}{l}\text { Medication Possession Ratio } \\
\text { (MPR). Patients were } \\
\text { considered adherent if } \\
\text { MPR } \geq 0.8\end{array}$ & $\begin{array}{l}\text { Decreased non-adherence: } \\
\text { older age, provider is a Family } \\
\text { Medicine Specialist Clinic, } \\
\text { more provider follow-up visits, } \\
\text { comorbidities }\end{array}$ \\
\hline
\end{tabular}

(Continued) 
Table 2 (Continued).

\begin{tabular}{|c|c|c|c|c|c|}
\hline Article & Study Design & Setting (Country) & $\begin{array}{l}\text { Patient } \\
\text { Population }\end{array}$ & Adherence Measure & $\begin{array}{l}\text { Determinants of Non- } \\
\text { Adherence }\end{array}$ \\
\hline $\begin{array}{l}\text { Wong et al } \\
(201 \mathrm{I})^{31}\end{array}$ & $\begin{array}{l}\text { Retrospective } \\
\text { cohort study }\end{array}$ & $\begin{array}{l}\text { Primary care clinic in I } \\
\text { territory of Hong Kong (China) }\end{array}$ & $\begin{array}{l}12.875 \\
\text { patients }\end{array}$ & $\begin{array}{l}\text { Discontinuation was } \\
\text { considered as the absence of } \\
\text { a refill prescription in all } \\
\text { subsequent clinic visits without } \\
\text { issuance of another lipid- } \\
\text { lowering agent } 180 \text { days from } \\
\text { the first prescription date }\end{array}$ & $\begin{array}{l}\text { Decreased non-adherence: } \\
\text { older age, male sex, fee payers, } \\
\text { provider is a Family Medicine } \\
\text { Specialist Clinic, residents in } \\
\text { rural districts, more provider } \\
\text { follow-up visits, comorbidities, } \\
\text { taking fibrates }\end{array}$ \\
\hline $\begin{array}{l}\text { Brogaard } \\
\text { et al } \\
(2012)^{32}\end{array}$ & $\begin{array}{l}\text { Retrospective } \\
\text { cohort study }\end{array}$ & $\begin{array}{l}\text { Department of Cardiology, } \\
\text { Odense University Hospital } \\
\text { (Denmark) }\end{array}$ & $\begin{array}{l}\text { Two } \\
\text { cohorts: } \\
\text { 2003: } 474 \\
\text { patients } \\
\text { 2008: } 550 \\
\text { patients }\end{array}$ & $\begin{array}{l}\text { Medication Possession Ratio } \\
\text { (MPR). Patients were } \\
\text { considered adherent if they } \\
\text { had an MPR } \geq 80 \%\end{array}$ & $\begin{array}{l}\text { Increased non-adherence: } \\
\text { younger age } \\
\text { Decreased non-adherence: } \\
\text { older age ( } \geq 80 \text { years) }\end{array}$ \\
\hline $\begin{array}{l}\text { Cheetham } \\
\text { et al } \\
(2013)^{33}\end{array}$ & $\begin{array}{l}\text { Retrospective } \\
\text { cohort study }\end{array}$ & $\begin{array}{l}\text { Kaiser Permanente Southern } \\
\text { California (KPSC), a managed } \\
\text { care organization (MCO), in } \\
\text { Southern California (USA) }\end{array}$ & $\begin{array}{l}19.826 \\
\text { patients }\end{array}$ & $\begin{array}{l}\text { Patients were followed for } \\
\text { a period of } 90 \text { days after the } \\
\text { index date. Patients who did } \\
\text { not pick up their new statin } \\
\text { prescriptions within this } 90 \text {-day } \\
\text { window were considered } \\
\text { primary nonadherent. }\end{array}$ & $\begin{array}{l}\text { Increased non-adherence: } \\
\text { African American ethnicity, } \\
\text { higher provider age, provider is } \\
\text { a high prescriber (>7 statin } \\
\text { prescriptions during study) } \\
\text { Decreased non-adherence: } \\
\text { older age, LDL cholesterol } \\
>160 \mathrm{mg} / \mathrm{dL} \text {, higher health care } \\
\text { utilization (ED visits, } \\
\text { hospitalizations, clinic visits or } \\
\text { any Rx, in a year), higher } \\
\text { Charlson Comorbidity Index, } \\
\text { provider is male }\end{array}$ \\
\hline $\begin{array}{l}\text { Christian } \\
\text { et al } \\
(2013)^{34}\end{array}$ & $\begin{array}{l}\text { Retrospective } \\
\text { cohort study }\end{array}$ & $\begin{array}{l}\text { US insurance-carrying } \\
\text { individuals (USA) }\end{array}$ & $\begin{array}{l}42.685 \\
\text { patients }\end{array}$ & $\begin{array}{l}\text { Adherence was quantified using } \\
\text { medication possession ratio } \\
\text { (MPR). Patients were } \\
\text { categorized into } 4 \mathrm{MPR} \\
\text { categories }(<0.40,0.40-0.59 \text {, } \\
0.60-0.79 \text {, and } \geq 0.80 \text { ), where } \\
80 \% \text { or greater is commonly } \\
\text { defined as adherent. }\end{array}$ & $\begin{array}{l}\text { Increased non-adherence: } \\
\text { female sex, peripheral vascular } \\
\text { disease, chronic pulmonary } \\
\text { disease, diabetes, liver disease, } \\
\text { rheumatic disease } \\
\text { Decreased non-adherence: } \\
\text { older age, initial treatment } \\
\text { drug being niacin + statin, } \\
\text { higher prescription drug } \\
\text { classes }\end{array}$ \\
\hline $\begin{array}{l}\text { Xie et al } \\
(2013)^{35}\end{array}$ & $\begin{array}{l}\text { Cross-sectional } \\
\text { study }\end{array}$ & $\begin{array}{l}\text { Twelve cities, and one county, } \\
\text { entailing } 2 \text { I province-level } \\
\text { hospitals (equivalent to } \\
\text { a teaching hospital in Europe) } \\
\text { and six county-level hospitals } \\
\text { (equivalent to a district hospital } \\
\text { in Europe) (China) }\end{array}$ & $\begin{array}{l}1.890 \\
\text { patients }\end{array}$ & $\begin{array}{l}\text { Medication Possession Ratio } \\
\text { (MPR). We defined good } \\
\text { compliance has an MPR of } \\
\geq 80 \%\end{array}$ & $\begin{array}{l}\text { Decreased non-adherence: } \\
\text { unemployed, cover rate of } \\
\text { medicine insurance } \geq 70 \% \text {, } \\
\text { Province/County hospital, } \\
\text { being attended in Cardiology } \\
\text { department, very high CVD } \\
\text { risk, using statins }\end{array}$ \\
\hline $\begin{array}{l}\text { Cicero et al } \\
(2014)^{36}\end{array}$ & $\begin{array}{l}\text { Retrospective } \\
\text { cohort study }\end{array}$ & $\begin{array}{l}\text { Lipid clinics of the University of } \\
\text { Bologna and the University of } \\
\text { Pavia (Italy) }\end{array}$ & 628 patients & $\begin{array}{l}\text { Persistence in medication after } \\
2 \text { years }\end{array}$ & $\begin{array}{l}\text { Decreased non-adherence: } \\
\text { female sex, family history of } \\
\text { early cardiovascular disease, } \\
\text { baseline LDL cholesterol, } \\
\text { treatment with nutraceuticals } \\
\text { versus statins }\end{array}$ \\
\hline
\end{tabular}

(Continued) 
Table 2 (Continued).

\begin{tabular}{|c|c|c|c|c|c|}
\hline Article & Study Design & Setting (Country) & $\begin{array}{l}\text { Patient } \\
\text { Population }\end{array}$ & Adherence Measure & $\begin{array}{l}\text { Determinants of Non- } \\
\text { Adherence }\end{array}$ \\
\hline $\begin{array}{l}\text { Halava et al } \\
(20 \mid 4)^{37}\end{array}$ & $\begin{array}{l}\text { Prospective cohort } \\
\text { study }\end{array}$ & $\begin{array}{l}\text { Data from the Finnish Public } \\
\text { Sector Study, a prospective } \\
\text { study involving public sector } \\
\text { employees in } 10 \text { municipalities } \\
\text { and } 21 \text { hospitals (Finland) }\end{array}$ & $\begin{array}{l}9.285 \\
\text { patients }\end{array}$ & $\begin{array}{l}\text { Proportion of days covered } \\
\text { (PDC). Non-adherence was } \\
\text { defined as PDC }<80 \%\end{array}$ & $\begin{array}{l}\text { Increased non-adherence: } \\
\text { female sex, younger age }(<60 \\
\text { years), single }\end{array}$ \\
\hline $\begin{array}{l}\text { Gaisenok } \\
\text { et al } \\
(2015)^{38}\end{array}$ & $\begin{array}{l}\text { Retrospective } \\
\text { observational study }\end{array}$ & $\begin{array}{l}\text { Preventive Pharmacotherapy } \\
\text { Department of the Ministry of } \\
\text { Healthcare of the Russian } \\
\text { Federation (Russia) }\end{array}$ & 274 patients & $\begin{array}{l}\text { Compliance with statin therapy } \\
\text { was assessed with information } \\
\text { on regularity of statin intake } \\
\text { and the responses to four } \\
\text { questions: (I) if they knew, } \\
\text { according to the results of } \\
\text { previous exams, that they had } \\
\text { elevated cholesterol levels; (2) } \\
\text { what method of } \\
\text { hypercholesterolemia } \\
\text { correction they used; (3) if } \\
\text { they were taking any statins; } \\
\text { and (4) if yes, what statin } \\
\text { preparation and what dose } \\
\text { they were taking. }\end{array}$ & $\begin{array}{l}\text { Increased non-adherence: } \\
\text { older age (>70 years) } \\
\text { Decreased non-adherence: } \\
\text { coronary heart disease, history } \\
\text { of myocardial infarction, } \\
\text { history of cardiovascular } \\
\text { intervention }\end{array}$ \\
\hline $\begin{array}{l}\text { Halava et al } \\
(2015)^{39}\end{array}$ & $\begin{array}{l}\text { Prospective cohort } \\
\text { study }\end{array}$ & $\begin{array}{l}\text { Retired, insured, swedish } \\
\text { patients taking statins (Sweden) }\end{array}$ & $\begin{array}{l}11.718 \\
\text { patients }\end{array}$ & $\begin{array}{l}\text { Adherence was defined as the } \\
\text { Proportion of Days Covered } \\
\text { (PDC). Non-adherence was } \\
\text { defined as (PDC }<80 \% \text { ). } \\
\text { Adherence was defined as } \\
\text { PDC } \geq 80 \%\end{array}$ & $\begin{array}{l}\text { Increased non-adherence: } \\
\text { retirement }\end{array}$ \\
\hline $\begin{array}{l}\text { Korhonen } \\
\text { et al } \\
(2015)^{40}\end{array}$ & $\begin{array}{l}\text { Prospective cohort } \\
\text { study }\end{array}$ & $\begin{array}{l}\text { Finnish Public Sector (10 towns } \\
\text { and } 6 \text { hospital districts) } \\
\text { (Finland) }\end{array}$ & $\begin{array}{l}1.916 \\
\text { patients }\end{array}$ & $\begin{array}{l}\text { Proportion of days covered } \\
\text { (PDC). Non-adherence was } \\
\text { defined as PDC }<80 \%\end{array}$ & $\begin{array}{l}\text { Increased non-adherence: } \\
\text { higher number of childhood } \\
\text { adversities (only in men), } \\
\text { experiencing severe conflicts in } \\
\text { the family }\end{array}$ \\
\hline $\begin{array}{l}\text { Warren et al } \\
(2015)^{4 l}\end{array}$ & $\begin{array}{l}\text { Linked data from } \\
\text { a prospective study }\end{array}$ & $\begin{array}{l}\text { Data from a prospective study } \\
\text { of } 267,09 \text { I Australians with age } \\
\geq 45 \text { years to national data sets } \\
\text { on prescription } \\
\text { reimbursements, general } \\
\text { practice claims, hospitalizations } \\
\text { and deaths (Australia) }\end{array}$ & $\begin{array}{l}36.144 \\
\text { patients }\end{array}$ & $\begin{array}{l}\text { Medication possession ratio } \\
\text { (MPR). Patients were } \\
\text { considered adherent if } \\
\text { MPR } \geq 80 \%\end{array}$ & $\begin{array}{l}\text { Increased non-adherence: } \\
\text { highest education qualification, } \\
\text { language spoken at home is } \\
\text { other than English, single, } \\
\text { widowed or separated, } \\
\text { employed, annual income } \geq \\
\$ 70.000 \text {, smoking habit, new } \\
\text { statin user, very high } \\
\text { psychological distress } \\
\text { Decreased non-adherence: } \\
\text { older age, private health } \\
\text { insurance, obesity, past smoker, } \\
\text { self-reported heart disease, } \\
\text { higher number of } \\
\text { comorbidities, living in more } \\
\text { remote areas }\end{array}$ \\
\hline
\end{tabular}

(Continued) 
Table 2 (Continued).

\begin{tabular}{|c|c|c|c|c|c|}
\hline Article & Study Design & Setting (Country) & $\begin{array}{c}\text { Patient } \\
\text { Population }\end{array}$ & Adherence Measure & $\begin{array}{c}\text { Determinants of Non- } \\
\text { Adherence }\end{array}$ \\
\hline $\begin{array}{l}\text { Korhonen } \\
\text { et al } \\
(2016)^{42}\end{array}$ & $\begin{array}{l}\text { Prospective cohort } \\
\text { study }\end{array}$ & $\begin{array}{l}\text { Employees of ten towns and six } \\
\text { hospital districts who were } \\
\text { targeted by questionnaire } \\
\text { surveys in } 2 / 4-\text { year intervals } \\
\text { since } 2000 \text { (Finland) }\end{array}$ & $\begin{array}{l}1.924 \\
\text { individuals }\end{array}$ & $\begin{array}{l}\text { Adherence was measured using } \\
\text { the Proportion of Days } \\
\text { Covered (PDC). Non- } \\
\text { adherence was defined as } \\
\text { PDC }<80 \%\end{array}$ & $\begin{array}{l}\text { Increased non-adherence: } \\
\text { depression, anxiety }\end{array}$ \\
\hline $\begin{array}{l}\text { Kronish et al } \\
(2016)^{43}\end{array}$ & $\begin{array}{l}\text { Retrospective } \\
\text { cohort study }\end{array}$ & $\begin{array}{l}\text { Centers for Medicare and } \\
\text { Medicaid Services Chronic } \\
\text { Condition Data Warehouse } \\
\text { (USA) }\end{array}$ & $\begin{array}{l}6.618 \\
\text { patients }\end{array}$ & $\begin{array}{l}\text { Proportion of days covered } \\
\text { (PDC). Patients were } \\
\text { categorized as nonadherent } \\
\text { (PDC }<80 \% \text { ) or adherent } \\
\text { (PDC } \geq 80 \% \text { ) }\end{array}$ & $\begin{array}{l}\text { Increased non-adherence: } \\
\text { older age (>75 years), diabetes } \\
\text { mellitus, depression, dementia } \\
\text { Decreased non-adherence: } \\
\text { being eligible for low-income } \\
\text { subsidy }\end{array}$ \\
\hline $\begin{array}{l}\text { Tokgözoğlu } \\
\text { et al } \\
(2016)^{44}\end{array}$ & $\begin{array}{l}\text { National cross- } \\
\text { sectional non- } \\
\text { interventional } \\
\text { observational study }\end{array}$ & $\begin{array}{l}\text { I4 centers of family medicine/ } \\
\text { general practice and cardiology } \\
\text { clinics (Turkey) }\end{array}$ & 532 patients & $\begin{array}{l}\text { Discontinuation of statin } \\
\text { therapy is defined as failure to } \\
\text { renew a statin prescription } \\
\text { with a } \geq 30 \text {-day gap between } \\
\text { the end of a prescription and } \\
\text { the start of the next } \\
\text { prescription }\end{array}$ & $\begin{array}{l}\text { Increased non-adherence: } \\
\text { higher educational status, } \\
\text { negative coverage of } \\
\text { information about statin } \\
\text { treatment and side effects } \\
\text { (hepatic, renal and muscular) } \\
\text { on television, patients' lack of } \\
\text { sufficient knowledge regarding } \\
\text { high cholesterol and related } \\
\text { risks }\end{array}$ \\
\hline $\begin{array}{l}\text { Al-Foraih } \\
\text { et al } \\
(2017)^{45}\end{array}$ & $\begin{array}{l}\text { Cross-sectional } \\
\text { study study }\end{array}$ & $\begin{array}{l}\text { Twelve polyclinic/general } \\
\text { practice clinics across } 4 \text { of the } \\
6 \text { governorates in Kuwait } \\
\text { (Kuwait) }\end{array}$ & 200 patients & $\begin{array}{l}\text { Adherence was assessed using } \\
\text { the 8-item self-report Morisky } \\
\text { Medication Adherence Scale } \\
\text { (MMAS-8) }\end{array}$ & $\begin{array}{l}\text { Increased non-adherence: } \\
\text { diabetes } \\
\text { Decreased non-adherence: } \\
\text { older age }\end{array}$ \\
\hline $\begin{array}{l}\text { Booth et al } \\
(2017)^{46}\end{array}$ & $\begin{array}{l}\text { Retrospective } \\
\text { cohort study }\end{array}$ & $\begin{array}{l}\text { Medicare beneficiaries with } \\
\text { a hospitalization for myocardial } \\
\text { infarction (USA) }\end{array}$ & $\begin{array}{l}158.795 \\
\text { patients }\end{array}$ & $\begin{array}{l}\text { Proportion of Days Covered } \\
\text { (PDC) High persistence was } \\
\text { defined as PDC } \geq 80 \%\end{array}$ & $\begin{array}{l}\text { Increased non-adherence: } \\
\text { not having a history of statin } \\
\text { use, others than non-Hispanic } \\
\text { white, cardiologist care prior } \\
\text { to myocardial infarction } \\
\text { hospitalization, diabetes } \\
\text { mellitus, history of chronic } \\
\text { kidney disease, heart failure, } \\
\text { coronary heart disease, statin } \\
\text { intolerance } \\
\text { Decreased non-adherence: } \\
\text { male sex, low-income subsidy, } \\
\text { higher area-level median } \\
\text { income, region of residence, } \\
\text { statin use prior to myocardial } \\
\text { infarction event, coronary } \\
\text { stent insertion, being } \\
\text { hospitalized for } \geq 5 \text { days, using } \\
\text { moderate-intensity and high- } \\
\text { intensity statins }\end{array}$ \\
\hline
\end{tabular}

(Continued) 
Table 2 (Continued).

\begin{tabular}{|c|c|c|c|c|c|}
\hline Article & Study Design & Setting (Country) & $\begin{array}{c}\text { Patient } \\
\text { Population }\end{array}$ & Adherence Measure & $\begin{array}{l}\text { Determinants of Non- } \\
\text { Adherence }\end{array}$ \\
\hline $\begin{array}{l}\text { Devaraj et al } \\
(2017)^{47}\end{array}$ & $\begin{array}{l}\text { Cross-sectional } \\
\text { study, by survey }\end{array}$ & $\begin{array}{l}\text { Urban primary care clinic } \\
\text { (Malaysia) }\end{array}$ & 398 patients & $\begin{array}{l}\text { 8-item Morisky Medication } \\
\text { Adherence Scale. A patient was } \\
\text { considered adherent if had } \\
\text { a score } \geq 6\end{array}$ & $\begin{array}{l}\text { Increased non-adherence: } \\
\text { male sex, duration of lipid } \\
\text { lowering therapy (>10 years), } \\
\text { taking medication(s) at night or } \\
\text { at no specific time, more } \\
\text { follow-up visits, single follow- } \\
\text { up clinic }\end{array}$ \\
\hline $\begin{array}{l}\text { Hickson } \\
\text { et al } \\
(2017)^{48}\end{array}$ & $\begin{array}{l}\text { Retrospective } \\
\text { cohort study }\end{array}$ & $\begin{array}{l}\text { Medicare beneficiaries taking } \\
\text { statins, with age } \geq 66 \text { years and } \\
\text { with an acute myocardial } \\
\text { infarction hospitalization in } \\
2008-2010 \text { (USA) }\end{array}$ & $\begin{array}{l}113.296 \\
\text { patients }\end{array}$ & $\begin{array}{l}\text { Proportion of days covered } \\
\text { (PDC). Patients were } \\
\text { categorized into } 3 \text { categories: } \\
\text { severely nonadherent (PDC } \\
<40 \% \text { ), moderately } \\
\text { nonadherent (PDC } 40-79.9 \% \text { ), } \\
\text { and adherent (PDC } \geq 80 \% \text { ) }\end{array}$ & $\begin{array}{l}\text { Increased non-adherence: } \\
\text { African American ethnicity, } \\
\text { Hispanic ethnicity, higher } \\
\text { baseline Charlson Comorbidity } \\
\text { Index, dementia/Alzheimer's } \\
\text { disease, depression, } \\
\text { revascularization/cardiac } \\
\text { intervention, ischemic heart } \\
\text { disease, rhabdomyolysis/ } \\
\text { myopathy, higher length of stay } \\
\text { in hospital post cardiovascular } \\
\text { event } \\
\text { Decreased non-adherence: } \\
\text { dual insurance eligibility } \\
\text { (Medicare and Medicaid), } \\
\text { follow-up care by Cardiologist } \\
\text { only }\end{array}$ \\
\hline $\begin{array}{l}\text { Wawruch } \\
\text { et al } \\
(2017)^{49}\end{array}$ & $\begin{array}{l}\text { Retrospective } \\
\text { cohort study }\end{array}$ & $\begin{array}{l}\text { The sample for this study was } \\
\text { assembled from the database of } \\
\text { the General Health Insurance } \\
\text { Company (Slovak Republic). }\end{array}$ & 797 patients & $\begin{array}{l}\text { Treatment gap was defined as } \\
\text { the presence of an at least } \\
\text { 6-month period without any } \\
\text { statin prescription. Patients } \\
\text { with a treatment gap period } \\
\text { were designated as 'non- } \\
\text { persistent', whereas those } \\
\text { without such a gap were } \\
\text { designated as 'persistent' } \\
\text { patients }\end{array}$ & $\begin{array}{l}\text { Increased non-adherence: } \\
\text { female sex } \\
\text { Decreased non-adherence: } \\
\text { older age ( } \geq 65 \text { years), } \\
\text { hypertension, diabetes mellitus, } \\
\text { hypercholesterolemia, higher } \\
\text { number of medications }\end{array}$ \\
\hline $\begin{array}{l}\text { Chung et al } \\
(2018)^{50}\end{array}$ & $\begin{array}{l}\text { Prospective, non- } \\
\text { interventional, } \\
\text { observational study }\end{array}$ & $\begin{array}{l}\text { Hospital Departments of } \\
\text { Neurology (Korea) }\end{array}$ & 991 patients & $\begin{array}{l}\text { Patient self-reported } \\
\text { adherence to statin therapy } \\
\text { was assessed using the Morisky } \\
\text { Medication Adherence Scale } \\
\text { (MMAS-8) }\end{array}$ & $\begin{array}{l}\text { Increased non-adherence: } \\
\text { taking high statin dose, higher } \\
\text { daily number of medication } \\
\text { pills } \\
\text { Decreased non-adherence: } \\
\text { patients' awareness of } \\
\text { hyperlipidemia, current drinker }\end{array}$ \\
\hline $\begin{array}{l}\text { do } \\
\text { Nascimento } \\
\text { et al } \\
(2018)^{51}\end{array}$ & $\begin{array}{l}\text { Cross-sectional } \\
\text { study, by survey }\end{array}$ & $\begin{array}{l}\text { I } 305 \text { primary healthcare } \\
\text { services located in } 272 \\
\text { Brazilian cities (Brazil) }\end{array}$ & $\begin{array}{l}8.803 \\
\text { patients }\end{array}$ & $\begin{array}{l}\text { Adherence was assessed by } \\
\text { asking patients whether they } \\
\text { missed any dose in the } 7 \text { days } \\
\text { prior to the interview. Patients } \\
\text { who reported missing at least } \\
\text { one dose in the past } 7 \text { days } \\
\text { were classified as having poor } \\
\text { adherence }\end{array}$ & $\begin{array}{l}\text { Decreased non-adherence: } \\
\text { older age, higher education, } \\
\text { dyslipidemia and diabetes } \\
\text { mellitus, heart diseases, } \\
\text { polypharmacy }(\geq 5) \text {, alcohol } \\
\text { use, not using emergency care }\end{array}$ \\
\hline
\end{tabular}

(Continued) 
Table 2 (Continued).

\begin{tabular}{|c|c|c|c|c|c|}
\hline Article & Study Design & Setting (Country) & $\begin{array}{c}\text { Patient } \\
\text { Population }\end{array}$ & Adherence Measure & $\begin{array}{l}\text { Determinants of Non- } \\
\text { Adherence }\end{array}$ \\
\hline $\begin{array}{l}\text { Haddad et al } \\
(2018)^{52}\end{array}$ & $\begin{array}{l}\text { Cross-sectional } \\
\text { study }\end{array}$ & $\begin{array}{l}20 \text { community pharmacies } \\
\text { from all } 5 \text { districts of Lebanon } \\
\text { (list provided by the Lebanese } \\
\text { Order of Pharmacists) } \\
\text { (Lebanon) }\end{array}$ & 247 patients & $\begin{array}{l}\text { Adherence was assessed using } \\
\text { a } 3 \text { questions questionnaire, } \\
\text { asking patients about the } \\
\text { frequency, percentage, and } \\
\text { rating response of their statin } \\
\text { use during previous month. } \\
\text { Possible responses were } \\
\text { divided in } 6 \text { categories: } 0 \% \text {, } \\
20 \%, 40 \%, 60 \%, 80 \% \text { and } 100 \% \text {. } \\
\text { A total score was calculated by } \\
\text { summing all } 3 \text { answers and } \\
\text { presented in a percentage. }\end{array}$ & $\begin{array}{l}\text { Increased non-adherence: } \\
\text { divorced, widowed, primary } \\
\text { and university level of } \\
\text { education, smoking } 15 \\
\text { cigarettes per day, not alcohol } \\
\text { drinkers, duration of } \\
\text { dyslipidemia } \\
\text { Decreased non-adherence: } \\
\text { married, single, secondary } \\
\text { educational level, non-smokers, } \\
\text { occasional alcohol drinking, } \\
\text { better illness perception, } \\
\text { better treatment satisfaction, } \\
\text { better quality of life perception }\end{array}$ \\
\hline $\begin{array}{l}\text { Kriegbaum } \\
\text { et al } \\
(2018)^{53}\end{array}$ & $\begin{array}{l}\text { Cross-sectional } \\
\text { study, by survey }\end{array}$ & $\begin{array}{l}\text { Danish population participating } \\
\text { in "LIFESTAT - Living with } \\
\text { Statins Project" (Denmark) }\end{array}$ & $\begin{array}{l}3.050 \\
\text { patients }\end{array}$ & $\begin{array}{l}\text { Discontinuation was based on } \\
\text { self-reporting of current statin } \\
\text { use in the interview, with } 3 \\
\text { possible predefined answers. } \\
\text { Discontinuation was defined as } \\
\text { those who answered: "I have } \\
\text { previously used cholesterol } \\
\text { lowering drugs" }\end{array}$ & $\begin{array}{l}\text { Increased non-adherence: } \\
\text { experience of side effects, fear } \\
\text { of side effects, feelings of } \\
\text { uncertainty and confusion } \\
\text { regarding information on } \\
\text { statins (from both mass media } \\
\text { and from general practitioners) }\end{array}$ \\
\hline $\begin{array}{l}\text { Ofori- } \\
\text { Asenso et al } \\
(2018)^{54}\end{array}$ & $\begin{array}{l}\text { Retrospective } \\
\text { cohort study }\end{array}$ & $\begin{array}{l}\text { A } 10 \% \text { random sample of the } \\
\text { Australian population from } \\
\text { data from the Pharmaceutical } \\
\text { Benefits Scheme (Australia) }\end{array}$ & $\begin{array}{l}49.380 \\
\text { patients }\end{array}$ & $\begin{array}{l}\text { The number of days on statin } \\
\text { were calculated, assuming } \\
\text { a dosage of I tablet daily. } \\
\text { Discontinuation was defined as } \\
\text { the first } \geq 90 \text { days without } \\
\text { statin coverage }\end{array}$ & $\begin{array}{l}\text { Increased non-adherence: } \\
\text { older age (>75 years), female } \\
\text { sex, therapeutic initiated by } \\
\text { general practitioner, diabetes } \\
\text { Decreased non-adherence: } \\
\text { taking atorvastatin or } \\
\text { rosuvastatin (VS simvastatin), } \\
\text { use of cardiovascular } \\
\text { pharmacotherapies } \\
\text { (antiplatelet, } \beta \text {-blockers, ACE } \\
\text { inhibitors or ARBs) }\end{array}$ \\
\hline $\begin{array}{l}\text { Chen et al } \\
(2019)^{55}\end{array}$ & $\begin{array}{l}\text { Population-based } \\
\text { retrospective } \\
\text { cohort study }\end{array}$ & $\begin{array}{l}\text { Taiwan National Health } \\
\text { Insurance claims (Taiwan) }\end{array}$ & $\begin{array}{l}169.624 \\
\text { patients }\end{array}$ & $\begin{array}{l}\text { Medication possession ratio } \\
\text { (MPR) and Proportion of days } \\
\text { covered (PDC). Good } \\
\text { adherence was considered if } \\
\text { PDC } \geq 0.80 \%\end{array}$ & $\begin{array}{l}\text { Increased non-adherence: } \\
\text { older age (>75 years), renal } \\
\text { disease, liver disease, } \\
\text { depression, chronic obstructive } \\
\text { pulmonary disease, taking } \\
\text { insulin, digitalis, OADs, ACEls or } \\
\text { diuretics } \\
\text { Decreased non-adherence: } \\
\text { female sex, previous statin user, } \\
\text { revascularization/cardiovascular } \\
\text { procedures, hyperlipidemia, } \\
\text { taking } \beta \text {-blockers, ARBs or } \\
\text { antiplatelet agents, healthcare } \\
\text { provider is a Medical Center, } \\
\text { statin prescribed by } \\
\text { Cardiovascular Medicine or } \\
\text { Cardiovascular Surgery }\end{array}$ \\
\hline
\end{tabular}

(Continued) 
Table 2 (Continued).

\begin{tabular}{|c|c|c|c|c|c|}
\hline Article & Study Design & Setting (Country) & $\begin{array}{c}\text { Patient } \\
\text { Population }\end{array}$ & Adherence Measure & $\begin{array}{l}\text { Determinants of Non- } \\
\text { Adherence }\end{array}$ \\
\hline $\begin{array}{l}\text { Phan et al } \\
(2019)^{56}\end{array}$ & $\begin{array}{l}\text { Retrospective } \\
\text { population-based } \\
\text { cohort study }\end{array}$ & $\begin{array}{l}\text { Integrated healthcare system in } \\
\text { Southern California (USA) }\end{array}$ & $\begin{array}{l}5.629 \\
\text { patients }\end{array}$ & $\begin{array}{l}\text { Proportion of Days Covered } \\
\text { (PDC). Adherence levels were } \\
\text { categorized as: high ( } \geq 80 \% \text { ), } \\
\text { partial ( } \geq 40 \% \text { and }<80 \%) \text {, and } \\
\text { low ( }<40 \%)\end{array}$ & $\begin{array}{l}\text { Increased non-adherence: } \\
\text { heart failure } \\
\text { Decreased non-adherence: } \\
\text { male sex, Caucasian, } \\
\text { hyperlipidemia, higher } \\
\text { adherence to } \beta \text {-blocker }\end{array}$ \\
\hline $\begin{array}{l}\text { Waßmuth } \\
\text { et al } \\
(2019)^{57}\end{array}$ & $\begin{array}{l}\text { Monocentric, } \\
\text { prospective cohort } \\
\text { study }\end{array}$ & $\begin{array}{l}\text { Department of Cardiology and } \\
\text { Department of Cardiac } \\
\text { Surgery of the University Clinic } \\
\text { Halle (Saale) (Germany) }\end{array}$ & 542 patients & $\begin{array}{l}\text { Adherence was defined has } \\
\text { unchanged if statins } \\
\text { and/or ezetimibe were } \\
\text { unchanged, newly prescribed, } \\
\text { or increased. Non-adherence } \\
\text { was considered if statins and/or } \\
\text { ezetimibe were stopped or } \\
\text { dosage was decreased }\end{array}$ & $\begin{array}{l}\text { Increased non-adherence: } \\
\text { peripheral artery disease, atrial } \\
\text { fibrillation, coronary artery } \\
\text { bypass surgery } \\
\text { Decreased non-adherence: } \\
\text { taking aspirin, } \mathrm{P} 2 \mathrm{Y} 12 \text { inhibitors } \\
\text { or } \beta \text {-blockers (at discharge } \\
\text { from cardiovascular event) }\end{array}$ \\
\hline $\begin{array}{l}\text { Bruckert } \\
\text { et al } \\
(2020)^{58}\end{array}$ & $\begin{array}{l}\text { Retrospective } \\
\text { observational } \\
\text { cohort study }\end{array}$ & $\begin{array}{l}\text { The Pharmacoepidemiologic } \\
\text { General Research eXtension } \\
\text { (PGRx)-acute coronary } \\
\text { syndrome (ACS) registry, from } \\
\text { cardiology centres (France) }\end{array}$ & $\begin{array}{l}2.695 \\
\text { patients }\end{array}$ & $\begin{array}{l}\text { Adherence was measured using } \\
\text { the Proportion of Days } \\
\text { Covered (PDC). Patients were } \\
\text { considered adherent if they } \\
\text { had a PDC } \geq 80 \%\end{array}$ & $\begin{array}{l}\text { Decreased non-adherence: } \\
\text { previous cardiovascular disease } \\
\text { event }\end{array}$ \\
\hline $\begin{array}{l}\text { Seaman et al } \\
(2020)^{59}\end{array}$ & $\begin{array}{l}\text { Retrospective } \\
\text { observational study }\end{array}$ & $\begin{array}{l}\text { Western Australian Population } \\
\text { (Australia) }\end{array}$ & $\begin{array}{l}205.924 \\
\text { patients }\end{array}$ & $\begin{array}{l}\text { Medication possession ratio } \\
\text { (MPR), with the threshold of } \\
\text { adherence defined as } 80 \%\end{array}$ & $\begin{array}{l}\text { Increased non-adherence: } \\
\text { younger age, living in more } \\
\text { remote areas, not having } \\
\text { previous ischemic heart } \\
\text { disease, not having previous } \\
\text { coronary artery } \\
\text { revascularization procedure, } \\
\text { not having previous statin use, } \\
\text { beneficiary status, not taking } \\
\text { cardiovascular medication, } \\
\text { higher number of other } \\
\text { medications } \\
\text { Decreased non-adherence: } \\
\text { older age }\end{array}$ \\
\hline $\begin{array}{l}\text { Shakarneh } \\
\text { et al } \\
(2020)^{60}\end{array}$ & $\begin{array}{l}\text { Cross-sectional } \\
\text { study, by survey }\end{array}$ & $\begin{array}{l}\text { Two middle governmental } \\
\text { primary healthcare clinics in } \\
\text { the cities of Ramallah and } \\
\text { Bethlehem (Palestine) }\end{array}$ & 185 patients & $\begin{array}{l}\text { Adherence was determined } \\
\text { using the 4-item Morisky } \\
\text { medication adherence scale } \\
\text { (MMAS-4). Total scores were } \\
\text { summed (range 0-4), with } \\
\text { scores of 0-I denoting high } \\
\text { adherence and 2-4 denoting } \\
\text { low adherence }\end{array}$ & $\begin{array}{l}\text { Increased non-adherence: } \\
\text { low education (illiterate), } \\
\text { polypharmacy (> 4), } \\
\text { comorbidities, concerns about } \\
\text { side effects }\end{array}$ \\
\hline
\end{tabular}

were retrospective, 12 cross-sectional studies and 9 prospective cohort studies. The patient's population in the studies ranged from 117 to 229.918 patients. In terms of measures of adherence, 15 studies used the "Proportion of Days Covered (PDC)", 7 used the "Medication Possession Ratio (MPR)", 3 used the "8-item self-report Morisky Medication Adherence Scale (MMAS-8)" and 2 used the "4-item self-report Morisky Medication Adherence Scale (MMAS-4)". The remaining 18 studies had a more specific measure of adherence, designed for each, individually. 


\section{Determinants for Non-Adherence to Medications}

We found several relevant determinants associated with non-adherence, summarized in Table 3.

\section{Sociodemographic Variables}

\section{Age}

Non-adherence decreases with age. ${ }^{16,26,33,45}$ For each 10year increase in age, the risk of non-adherence decreases about 5-11\%. ${ }^{23,27}$ Analyzing particular age groups, we observed the same trend of decreasing non-adherence with higher age. Non-adherence is higher in younger patients (age $\leq 50$ years $),{ }^{14,32,37,59}$ reaching values of $50 \%{ }^{20}$ It is lower between 50 and 70 years $(8-34 \%),{ }^{17,19,20,28,30,31,41}$ and in older patients (age $\geq 70$ years), ${ }^{25,30,31,59}$ who present the risk of non-adherence of $11-65 \%$. $^{17,28,41,49}$

\section{Sex}

In general, females are more non-adherent than males, ${ }^{26,31,34,37,46,56}$ with an increased risk of 5$31 \%$. $^{17,20,23,27,49,54}$

Table 3 Main Determinants Associated with Non-Adherence

\begin{tabular}{|c|c|c|}
\hline \multicolumn{3}{|c|}{ Main Identified Determinants Associated with Non-Adherence } \\
\hline & Associated with Higher Non-Adherence & Associated with Lower Non-Adherence \\
\hline Age & Younger age ( $\leq 50$ years) & Older age \\
\hline Sex & Female sex & Male sex \\
\hline Ethnicity & $\begin{array}{l}\text { African American } \\
\text { Hispanic }\end{array}$ & Caucasian \\
\hline Marital Status & $\begin{array}{l}\text { Divorced } \\
\text { Separated } \\
\text { Widowed } \\
\text { Single }\end{array}$ & Married \\
\hline Habits & Smoking habits & Alcohol drinking habits \\
\hline Comorbidities & $\begin{array}{l}\text { Chronic obstructive pulmonary disease } \\
\text { Alzheimer disease/dementia } \\
\text { Depression } \\
\text { Diabetes } \\
\text { Anxiety } \\
\text { Hepatic disease } \\
\text { Chronic kidney disease } \\
\text { Peripheral vascular disease } \\
\text { Heart failure }\end{array}$ & $\begin{array}{l}\text { Higher number of comorbidities } \\
\text { Higher number of cardiovascular risk factors } \\
\text { Present heart disease } \\
\text { Hypertension }\end{array}$ \\
\hline Medications & $\begin{array}{l}\text { New users of lipid-lowering medications } \\
\text { Patients presenting side effects or concerns } \\
\text { about them } \\
\text { Higher duration of lipid-lowering treatment }\end{array}$ & $\begin{array}{l}\text { Patients taking } \beta \text {-blockers } \\
\text { Patients taking antiplatelets }\end{array}$ \\
\hline $\begin{array}{l}\text { Cardiovascular diseases and } \\
\text { interventions }\end{array}$ & & $\begin{array}{l}\text { History of cardiovascular disease events } \\
\text { History of cardiac intervention or revascularization } \\
\text { procedure }\end{array}$ \\
\hline Healthcare services utilization & & $\begin{array}{l}\text { Higher healthcare services utilization } \\
\text { Patients treated in cardiology } \\
\text { Patients treated in family medicine } \\
\text { Having health insurance } \\
\text { Higher number of follow-up visits }\end{array}$ \\
\hline Other determinants & $\begin{array}{l}\text { Unemployment } \\
\text { Bad doctor-patient relationship }\end{array}$ & \\
\hline
\end{tabular}




\section{Ethnicity}

Several studies show an increase of non-adherence in African American patients, ${ }^{33,48}$ up to $30 \%{ }^{23}$ Hispanics $^{23,48}$ are also associated with worst adherence to medications.

\section{Marital Status}

Non-adherence is lower in married patients, ${ }^{52}$ higher in single patients ${ }^{37,41}$ and increased in divorced, separated or widowed patients. ${ }^{41,52}$

\section{Employment Status}

Non-adherence is worst in unemployed. ${ }^{35,41}$

\section{Lifestyle Variables}

\section{Smoking Status}

Non-adherence is higher in current smokers, ${ }^{14,41,52}$ with an increase of $18-163 \% .{ }^{17,21}$

\section{Alcohol Drinking Status}

Non-adherence is lower in active alcohol drinkers, ${ }^{52}$ showing values of odds ratio between 0.61 and $0.71 .^{50,51}$

\section{Medication Use}

Several conditions regarding medications are associated with non-adherence. In general, non-adherence is higher in new users of lipid-lowering medications, ${ }^{20,46,55,59}$ justifying up to $47 \%$ more risk. ${ }^{41}$ Also, in patients presenting side effects or expressing concerns about them, nonadherence is higher, ${ }^{14,24,53}$ with an associated odds ratio of 2.89. ${ }^{60}$ Non-adherence is also worst in patients having a higher duration of lipid-lowering treatment. ${ }^{19,47}$ On the other hand, several studies have shown that non-adherence is lower in patients taking $\beta$-blockers, ${ }^{55-57}$ who have approximately less $12 \%$ risk of non-adherence ${ }^{27,54}$ as in patients taking other cardiovascular medications. ${ }^{17,24,59}$

\section{Comorbidities/Disease Status}

\section{Number of Patients' Comorbidities}

Non-adherence decreases with a higher number of comorbidities. For each comorbidity present, the risk of non-adherence decreases by $4 \%{ }^{23}$ This is apparent since the first comorbidity, ${ }^{30,31,41}$ leading to $5 \%$ improvement. ${ }^{41}$ This is even more apparent in patients with two or more comorbidities, ${ }^{30,31,41}$ with a $7-8 \%$ improvement in adherence. ${ }^{41}$ Inversely, the absence of concomitant diseases is associated with a $59 \%$ increase in the risk of nonadherence. $^{21}$

\section{Type of Comorbidity}

Several comorbidities are identified as especially associated with higher non-adherence: chronic obstructive pulmonary disease, ${ }^{34,55}$ with a $7-10 \%$ increased risk; ${ }^{27}$ Alzheimer's disease or other dementias, ${ }^{48}$ with a $20 \%$ increased risk ${ }^{27,43}$ depression, ${ }^{18,26,48,55}$ with a $9-15 \%$ increased risk; $;^{42,43}$ diabetes, ${ }^{34,45,46}$ with a $7-10 \%$ increased risk of non-adherence ${ }^{43,54}$ anxiety; ${ }^{18,42}$ hepatic disease $;{ }^{34,55}$ chronic kidney disease $;{ }^{46,55}$ peripheral vascular disease ${ }^{34,57}$ and heart failure. ${ }^{46,56}$

On the other hand, non-adherence is lower in patients with present heart diseases, ${ }^{41,51}$ in patients with a higher number of cardiovascular risk factors, ${ }^{29,35}$ in patients with hypertension ${ }^{27,49}$ and in patients taking antiplatelets. ${ }^{54,55}$

\section{Cardiovascular Diseases and Interventions}

The history of cardiovascular disease events is associated with lower non-adherence. ${ }^{38,58,59}$ Patients with a previous cardiac intervention or a revascularization procedure, present lower non-adherence, ${ }^{46,59}$ with odds ratio of 0.19 0.68. ${ }^{19,38,55}$ Patients without a previous atherosclerotic event present $135 \%$ more risk of non-adherence. ${ }^{21}$

\section{Healthcare Related Variables}

\section{Healthcare Services Utilization}

Non-adherence is decreased in patients with higher healthcare services utilization. ${ }^{27,33}$ It is also influenced by providers, with cardiology $y^{35,48}$ and family medicine ${ }^{30,31}$ contributing for lower levels of non-adherence.

\section{Having Health Insurance}

Non-adherence is lower in patients who had health insurance. $^{35,48}$ These patients have 3-5\% less risk of nonadherence. $^{41}$

\section{Follow-Up Visits}

The follow-up visits are associated with lower nonadherence, ${ }^{24,30,31}$ with an impact on the risk of $60 \% .{ }^{17}$

\section{Physician-Patient Relationship}

Having a bad doctor-patient relationship ${ }^{14,24}$ is associated with increased non-adherence.

\section{Discussion}

Adherence is a cornerstone of the effectiveness of the medications. The best therapeutic will never work if the patient does not take it. In this review, we identify a set of determinants related to non-adherence to medications in patients with dyslipidemia. Although the different weight, 
each characteristic is associated with a significant variation in non-adherence, allowing to adjust and to personalize the therapeutic decision in these patients. The more of these conditions the higher should be our concern about the risk of non-adherence in the patient.

Our review included studies related to dyslipidemia, in a broader approach, to find a wide range of factors related to adherence. Our results are in line with other studies. OforiAsenso et al published a systematic review with metaanalysis, focused on the non-adherence to statins in patients aged $\geq 65$ years. ${ }^{61}$ It included 45 articles and identified the female sex, African American ethnicity, new lipid-lowering drug users, taking lower number of cardiovascular medications and having depression as factors associated with higher non-adherence. Contrary to our findings, this study identified kidney disease as a factor for decreased nonadherence. Mann et al also reviewed 22 cohort studies with meta-analysis, focusing on non-adherence to statins. He described a U-shaped association with age, higher in the younger and in the elderly and lower in middle ages, as in patients with diabetes. ${ }^{62}$ Unlike other reviews, we focused on patients with dyslipidemia, irrespective of the medication, and tried obtaining practical and objective information that could allow physicians to rapidly consult that information in a context of a consultation, namely on the visit where the first prescription occurs.

A difficulty in our review was the differences observed between the studies: the different study designs; different sample sizes of study population; different tools to measure adherence ("Proportion of Days Covered (PDC)", "Medication Possession Ratio (MPR)", the "8-item selfreport Morisky Medication Adherence Scale (MMAS-8)", the "4-item self-report Morisky Medication Adherence Scale (MMAS-4)"); different outcomes (many evaluated non-adherence, but others evaluated adherence, discontinuation, persistence); and the way to quantify them (odds ratio, risk ratio, hazard ratio, or a simple qualitative assessment). This heterogeneity does not seem to be enough to condition our results, as more than the quantification of non-adherence, it was more important to identify their causes. Some factors that our review does not account for are the impact of media and more precisely social media platforms. In fact, in today's society, these platforms of social interaction are of the utmost relevance as, in our view, they can contribute to changes in patients' perceptions and preconceptions regarding medications. We think that this reality possibly negatively influences patients' medical literacy and could, in most cases, create misconceptions and negatively affect patients' motivation to adhere to medications, undermining the importance of adherence.

This review allows to draw the profile of the nonadherent patient, to whom we should pay special attention and a hard intervention to lead them to take the lipidlowering medications appropriately, in their own benefit. Several strategies help to enhance the adherence to medications. ${ }^{63}$ First of all, it is really important that healthcare providers can detect non-adherence. This should be a systematic practice in every visit, in every patient, to quantify the problem and to register it in the clinical file. There are many ways to do it, both directly (counting pills or renewal of the prescriptions) or indirectly (by questionnaire or by comparison with the expected effect). Then, we must think about the best simplification of the regimen (using longer acting drugs or pills combing several drugs, adapting drug regimen to patients' daily activities and preferences, and thoroughly introducing the therapeutic regimen, gradually increasing complexity). Literacy plays a major role. ${ }^{64}$ Patients need to be aware of their disease and the associated risks. We must be sure that they, and most of times also their families, understand reasonably the instructions and have enough time to express their expectations, their fears and doubts: ${ }^{65}$ using a simple and clear language; writing the instructions and letting the patient to repeat them; giving the patient several options, if possible. Communication skills are crucial, as it is a good doctorpatient relationship and the continuity of care, creating a trustful environment and empathy. The strengthened therapeutic alliance facilitates the sharing of responsibility toward health, where physicians and patients play their own specific role. Time is also a major factor to consider, as it is increasingly scarce in nowadays medical practice. Indeed, all the above items require time: time investment in the visit, time to listen to the patients and time to establish a real and meaningful doctor-patient relationship.

This review provides a synthesis of the determinants for non-adherence in patients with dyslipidemia in a practical perspective. Our results allow the physicians to identify the factors in each patient and adjust their intervention to better deal with the risk of non-adherence in a preventive approach. With this information, the healthcare providers may better estimate the probability of non-adherence of the patient since the initial evaluation. Patients with higher probability of non-adherence would benefit from earlier and more robust approach to minimize the risk of not taking the medications. Logically, being 
a qualitative assessment, this probability is subjective and is highly dependent on the physician expertise and experience to evaluate the patient in each visit. Perhaps more work to further assess the determinants of non-adherence and the posterior development of a score based on that information could allow a more objective approach to these situations, further facilitating the systematization of this practice of evaluation of non-adherence profiles.

In the future, more research is necessary to further understand the weight of the different aspects and the way they interact with each other, including the patients' preferences, expectations and perceptions. More than a disease, dyslipidemia is a cardiovascular risk factor. Its treatment is mainly a preventive approach, and the efficacy is the non-occurrence of the event. This is very different from myocardial infarction, stroke or heart failure, where every measure presents a quick effect, measurable by the patient's own perception. In fact, patient's perception of the importance of adherence in dyslipidemia, along with the associated potential benefit, is a fundamental part of the problem that we ought to collectively improve.

\section{Conclusion}

There are important identifiable determinants of nonadherence in patients with dyslipidemia. Healthcare providers should identify such characteristics to approach patients in a personalized way, investing more time in improving adherence. These patients benefit from a specific approach to minimize the problem of nonadherence and improve the therapeutic success, improve health and save lives.

\section{Disclosure}

The authors report no conflict of interests in this work.

\section{References}

1. Yusuf S, Hawken S, Ounpuu S, et al. Effect of potentially modifiable risk factors associated with myocardial infarction in 52 countries (the INTERHEART study): case-control study. Lancet. 2004;364 (9438):937-952. doi:10.1016/S0140-6736(04)17018-9

2. Townsend N, Wilson L, Bhatnagar P, Wickramasinghe K, Rayner M, Nichols M. Cardiovascular disease in Europe: epidemiological update 2016. Eur Heart J. 2016;37(42):3232-3245. doi:10.1093/eurheartj/ ehw334

3. Catapano AL, Graham I, De Backer G, et al. 2016 ESC/EAS Guidelines for the Management of Dyslipidaemias: the Task Force for the Management of Dyslipidaemias of the European Society of Cardiology (ESC) and European Atherosclerosis Society (EAS) Developed with the special contribution of the European Association for Cardiovascular Prevention \& Rehabilitation (EACPR). Eur Heart J. 2016;2999-3058. doi:10.1093/eurheartj/ehw272
4. Sabaté E; World Health Organization. Adherence to Long-Term Therapies: Evidence for Action. Vol. xv. World Health Organization; 2003:198. Available from: http://apps.who.int/iris/bit stream/handle/10665/42682/9241545992.pdf;jsessionid= 28AA4EDC4BD4B97052696A4F4990D5D0? sequence $=1$. Accessed Aug 17, 2021.

5. Osterberg L, Blaschke T. Adherence to medication. Review. $N$ Engl $J$ Med. 2005;353(5):487-497. doi:10.1056/NEJMra050100

6. Naderi SH, Bestwick JP, Wald DS. Adherence to drugs that prevent cardiovascular disease: meta-analysis on 376,162 patients. Am J Med. 2012;125(9):882-7.e1. doi:10.1016/j.amjmed.2011.12.013

7. Cutler RL, Fernandez-Llimos F, Frommer M, Benrimoj C, GarciaCardenas V. Economic impact of medication non-adherence by disease groups: a systematic review. BMJ Open. 2018;8(1):e016982. doi:10.1136/bmjopen-2017-016982

8. De Vera MA, Bhole V, Burns LC, Lacaille D. Impact of statin adherence on cardiovascular disease and mortality outcomes: a systematic review. Br J Clin Pharmacol. 2014;78(4):684-698. doi:10.1111/bcp.12339

9. Bitton A, Choudhry NK, Matlin OS, Swanton K, Shrank WH. The impact of medication adherence on coronary artery disease costs and outcomes: a systematic review. Am J Med. 2013;126(4):357.e7-357. e27. doi:10.1016/j.amjmed.2012.09.004

10. Chowdhury R, Khan H, Heydon E, et al. Adherence to cardiovascular therapy: a meta-analysis of prevalence and clinical consequences. Eur Heart J. 2013;34(38):2940-2948. doi:10.1093/eurheartj/eht295

11. Carvalho AS, Santos P. Medication adherence in patients with arterial hypertension: the relationship with healthcare systems' organizational factors. Original. Patient Prefer Adherence. 2019;13:1761-1774. doi:10.2147/PPA.S216091

12. Moher D, Liberati A, Tetzlaff J, Altman DG. Preferred reporting items for systematic reviews and meta-analyses: the PRISMA statement. PLoS Med. 2009;6(7):e1000097. doi:10.1371/journal.pmed.1000097

13. National Heart Lung and Blood Institute. Quality Assessment Tool for Observational Cohort and Cross-Sectional Studies [internet]. National Institutes of Health; 2021 [cited 2021 Mar 31] Available from: https://www.nhlbi.nih.gov/health-topics/study-quality-assess ment-tools. Accessed August 23, 2021.

14. Kiortsis DN, Giral P, Bruckert E, Turpin G. Factors associated with low compliance with lipid-lowering drugs in hyperlipidemic patients. $J$ Clin Pharm Ther. 2000;25(6):445-451. doi:10.1046/j.13652710.2000.00315.x

15. Mansur AP, Mattar APL, Tsubo CE, Simão DT, Yoshi FR, Daci K. Prescription and adherence to statins of patients with coronary artery disease and hypercholesterolemia. Article. Arq Bras Cardiol. 2001;76 (2):111-118. doi:10.1590/S0066-782X2001000200002

16. O'Donnell DC, Brown CM, Piziak VK. Alternative therapy use and adherence to antihyperlipidemic drugs in a lipid clinic. Note. Ame J Health Syst Pharm. 2001;58(11):1017-1021. doi:10.1093/ajhp/58.11.1017

17. Yang CC, Jick SS, Testa MA. Discontinuation and switching of therapy after initiation of lipid-lowering drugs: the effects of comorbidities and patient characteristics. Br J Clin Pharmacol. 2003;56 (1):84-91. doi:10.1046/j.1365-2125.2003.01818.x

18. Stilley CS, Sereika S, Muldoon MF, Ryan CM, Dunbar-Jacob J. Psychological and cognitive function: predictors of adherence with cholesterol lowering treatment. Ann Behav Med. 2004;27 (2):117-124. doi:10.1207/s15324796abm2702_6

19. Benner JS, Pollack MF, Smith TW, Bullano MF, Willey VJ, Williams SA. Association between short-term effectiveness of statins and long-term adherence to lipid-lowering therapy. Am J Health Syst Pharm. 2005;62(14):1468-1475. doi:10.2146/ajhp040419

20. Caspard H, Chan AK, Walker AM. Compliance with a statin treatment in a usual-care setting: retrospective database analysis over 3 years after treatment initiation in health maintenance organization enrollees with dyslipidemia. Clin Ther. 2005;27(10):1639-1646. doi:10.1016/j.clinthera.2005.10.005 
21. Di Martino M, Degli Esposti L, Ruffo P, et al. Underuse of lipid-lowering drugs and factors associated with poor adherence: a real practice analysis in Italy. Eur J Clin Pharmacol. 2005;61 (3):225-230. doi:10.1007/s00228-005-0911-z

22. Huser MA, Evans TS, Berger V. Medication adherence trends with statins. Article. Adv Ther. 2005;22(2):163-171. doi:10.1007/BF02849887

23. Batal HA, Krantz MJ, Dale RA, Mehler PS, Steiner JF. Impact of prescription size on statin adherence and cholesterol levels. $B M C$ Health Serv Res. 2007;7:175. doi:10.1186/1472-6963-7-175

24. McGinnis B, Olson KL, Magid D, et al. Factors related to adherence to statin therapy. Ann Pharmacother. 2007;41(11):1805-1811. doi:10.1345/aph.1K209

25. Natarajan N, Putnam RW, Yip AM, Frail D. Family practice patients' adherence to statin medications. Can Fam Physician. 2007;53 (12):2144-2145.

26. Ye X, Gross CR, Schommer J, Cline R, St. Peter WL. Association between copayment and adherence to statin treatment initiated after coronary heart disease hospitalization: a longitudinal, retrospective, cohort study. Article. Clin Ther. 2007;29(12):2748-2757. doi:10.10 16/j.clinthera.2007.12.022

27. Chodick G, Shalev V, Gerber Y, et al. Long-term persistence with statin treatment in a not-for-profit health maintenance organization: a population-based retrospective cohort study in Israel. Article. Clin Ther. 2008;30(11):2167-2179. doi:10.1016/j.clinthera.2008.11.012

28. Vinker S, Shani M, Baevsky T, Elhayany A. Adherence with statins over 8 years in a usual care setting. Am J Manag Care. 2008;14 (6):388-392.

29. Latry P, Molimard M, Dedieu B, Couffinhal T, Bégaud B, MartinLatry K. Adherence with statins in a real-life setting is better when associated cardiovascular risk factors increase: a cohort study. BMC Cardiovasc Disord. 2011;11:46. doi:10.1186/1471-2261-11-46

30. Wong MC, Jiang JY, Griffiths SM. Adherence to lipid-lowering agents among 11,042 patients in clinical practice. Int J Clin Pract. 2011;65(7):741-748. doi:10.1111/j.1742-1241.2011.02706.x

31. Wong MCS, Jiang JY, Yan BP, Griffiths SM. Subjects at risk of discontinuation of lipid-lowering agents: a 6-month cohort study among 12,875 patients in a Chinese population. Article. Clin Ther. 2011;33(5):617-628. doi:10.1016/j.clinthera.2011.04.019

32. Brogaard HV, Køhn MG, Berget OS, et al. Significant improvement in statin adherence and cholesterol levels after acute myocardial infarction. Dan Med J. 2012;59(9):A4509.

33. Cheetham TC, Niu F, Green K, et al. Primary nonadherence to statin medications in a managed care organization. J Manag Care Pharm. 2013;19(5):367-373. doi:10.18553/jmcp.2013.19.5.367

34. Christian JB, Rabatin V, Lowe KA. Adherence, persistence, and predictors associated with early niacin termination. Article. J Pharm Technol. 2013;29(4):178-185. doi:10.1177/875512251302900406

35. Xie G, Zaman MJ, Myint PK, Liang L, Zhao L, Wu Y. Factors associated with compliance to lipid-lowering treatment in China. Eur J Prev Cardiol. 2013;20(2):229-237. doi:10.1177/2047487312438847

36. Cicero AF, Derosa G, Parini A, Baronio C, Borghi C. Factors associated with 2-year persistence in fully non reimbursed lipid-lowering treatments. Atherosclerosis. 2014;235(1):81-83. doi:10.1016/j. atherosclerosis.2014.04.016

37. Halava H, Korhonen MJ, Huupponen R, et al. Lifestyle factors as predictors of nonadherence to statin therapy among patients with and without cardiovascular comorbidities. Article. CMAJ. 2014;186(12): E449-E456. doi:10.1503/cmaj.131807

38. Gaisenok O, Martsevich S, Tripkosh S, Lukina Y. Analysis of lipid-lowering therapy and factors affecting regularity of statin intake in patients with cardiovascular disease enrolled in the PROFILE registry. Rev Port Cardiol. 2015;34(2):111-116. doi:10.1016/j. repc. 2014.08 .021
39. Halava H, Westerlund $H$, Korhonen MJ, et al. Influence of retirement on adherence to statins in the insurance medicine all-Sweden total population data base. PLoS One. 2015;10(6):e0130901. doi:10.1371/ journal.pone.0130901

40. Korhonen MJ, Halonen JI, Brookhart MA, et al. Childhood adversity as a predictor of non-adherence to statin therapy in adulthood. PLoS One. 2015;10(5):e0127638. doi:10.1371/journal.pone.0127638

41. Warren JR, Falster MO, Tran B, Jorm L. Association of continuity of primary care and statin adherence. PLoS One. 2015;10(10):e0140008. doi:10.1371/journal.pone.0140008

42. Korhonen MJ, Pentti J, Hartikainen J, Kivimäki M, Vahtera J. Somatic symptoms of anxiety and nonadherence to statin therapy. Int J Cardiol. 2016;214:493-499. doi:10.1016/j.ijcard.2016.04.003

43. Kronish IM, Ross JS, Zhao H, Muntner P. Impact of hospitalization for acute myocardial infarction on adherence to statins among older adults. Circ Cardiovasc Qual Outcomes. 2016;9(4):364-371. doi:10.1161/circoutcomes.115.002418

44. Tokgözoğlu L, Özdemir R, Altındağ R, et al. Patient characteristics and statin discontinuation-related factors during treatment of hypercholesterolemia: an observational non-interventional study in patients with statin discontinuation (STAY study). Turk Kardiyol Dern Ars. 2016;44(1):53-64. doi:10.5543/tkda.2015.47041

45. Al-Foraih M, Somerset S. Factors affecting adherence to statins in hypercholesterolemic Kuwaiti patients: a Cross-Sectional Study. Med Princ Pract. 2017;26(1):35-40. doi:10.1159/000450644

46. Booth JN 3rd, Colantonio LD, Chen L, et al. Statin discontinuation, reinitiation, and persistence patterns among medicare beneficiaries after myocardial infarction: a Cohort Study. Circ Cardiovasc Qual Outcomes. 2017;10(10):e003626. doi:10.1161/circoutcomes.117.003626

47. Devaraj NK, Mohamed M, Hussein N. Prevalence, factors influencing and knowledge about adherence to lipid-lowering therapy among hyperlipidemia patients. Med J Malaysia. 2017;72(3): 157-164.

48. Hickson RP, Robinson JG, Annis IE, et al. Changes in statin adherence following an acute myocardial infarction among older adults: patient predictors and the association with follow-up with primary care providers and/or cardiologists. J Am Heart Assoc. 2017;6(10): e007106. doi:10.1161/jaha.117.007106

49. Wawruch M, Zatko D, Wimmer G Jr, et al. Age-related differences in non-persistence with statin treatment in patients after a transient ischaemic attack. Clin Drug Investig. 2017;37(11):1047-1054. doi:10.1007/s40261-017-0559-3

50. Chung PW, Yoon BW, Lee YB, et al. Medication adherence of statin users after acute ischemic stroke. Eur Neurol. 2018;80(1-2):106-114. doi: $10.1159 / 000493530$

51. Do Nascimento R, Guerra AA Jr, Alvares J, et al. Statin use in Brazil: findings and implications. Curr Med Res Opin. 2018;34 (10):1809-1817. doi:10.1080/03007995.2018.1451312

52. Haddad C, Hallit S, Salhab M, et al. Association between adherence to statins, illness perception, treatment satisfaction, and quality of life among Lebanese patients. Article. J Cardiovasc Pharmacol Ther. 2018;23(5):414-422. doi:10.1177/1074248418769635

53. Kriegbaum M, Lau SR. Medication non-adherence and uncertainty: information-seeking and processing in the Danish LIFESTAT survey. Res Social Adm Pharm. 2018;14(8):736-741. doi:10.1016/j.sapharm. 2017.09.002

54. Ofori-Asenso R, Ilomaki J, Tacey M, et al. Switching, discontinuation, and reinitiation of statins among older adults. $J$ Am Coll Cardiol. 2018;72(21):2675-2677. doi:10.1016/j.jacc.2018.08.2191

55. Chen ST, Huang ST, Shau WY, et al. Long-term statin adherence in patients after hospital discharge for new onset of atherosclerotic cardiovascular disease: a population-based study of real world prescriptions in Taiwan. BMC Cardiovasc Disord. 2019;19(1):62. doi:10.1186/s12872-019-1032-4 
56. Phan DQ, Duan L, Lam B, et al. Statin adherence and mortality in patients aged 80 years and older after acute myocardial infarction. J Am Geriatr Soc. 2019;67(10):2045-2049. doi:10.1111/jgs.16037

57. Waßmuth S, Rohe K, Noack F, Noutsias M, Treede H, Schlitt A. Adherence to lipid-lowering therapy in patients with coronary heart disease from the state of saxony-anhalt, Germany. Article. Vasc Health Risk Manag. 2019;15:477-483. doi:10.2147/VHRM. S197089

58. Bruckert E, Desamericq G, Khachatryan A, Ngo P, Gusto G, Sorio-Vilela F. Patient characteristics, treatment patterns, and adherence to lipid-lowering therapies following an acute coronary syndrome. Rev Cardiovasc Med. 2020;21(4):643-650. doi:10.31083/j.rcm.2020.04.189

59. Seamon K, Sanfilippo F, Bulsara M, et al. Predictors of ceasing or reducing statin medication following a large increase in the consumer copayment for medications: a retrospective observational study. Public Health Res Pract. 2020;30(1):1-9. doi:10.17061/phrp29121905

60. Shakarneh JK, Hallak HO, Awadallah HB, Al-Hamed DH, Khdour MR. Necessity and concerns about lipid-lowering medical treatments and risk factors for non-adherence: a cross-sectional study in Palestine. Int J Clin Pract. 2020;74(7):e13511. doi:10.1111/ijcp.13511
61. Ofori-Asenso R, Jakhu A, Curtis AJ, et al. A systematic review and meta-analysis of the factors associated with nonadherence and discontinuation of statins among people aged $\geq 65$ years. J Gerontol A Biol Sci Med Sci. 2018;73(6):798-805. doi:10.1093/gerona/glx256

62. Mann DM, Woodward M, Muntner P, Falzon L, Kronish I. Predictors of nonadherence to statins: a systematic review and meta-analysis. Ann Pharmacother. 2010;44(9):1410-1421. doi:10. 1345/aph.1P150

63. Atreja A, Bellam N, Levy SR. Strategies to enhance patient adherence: making it simple. MedGenMed. 2005;7(1):4.

64. Santos P. Challenge of health literacy in cardiovascular disease prevention. Curr Res Cardiol. 2018;5(special issue 4):18.

65. Santos P, Nazare I, Martins C, Sa L, Couto L, Hespanhol A. As Normas de Orientacao Clinica em Portugal e os Valores dos Doentes. [The Portuguese Guidelines and Patients Values]. Acta Med Port. 2015;28(6):754-759. doi:10.20344/ amp. 6301
Patient Preference and Adherence

\section{Publish your work in this journal}

Patient Preference and Adherence is an international, peer-reviewed, open access journal that focusing on the growing importance of patient preference and adherence throughout the therapeutic continuum. Patient satisfaction, acceptability, quality of life, compliance, persistence and their role in developing new therapeutic modalities and compounds to optimize clinical outcomes for existing disease

\section{Dovepress}

states are major areas of interest for the journal. This journal has been accepted for indexing on PubMed Central. The manuscript management system is completely online and includes a very quick and fair peer-review system, which is all easy to use. Visit http:// www.dovepress.com/testimonials.php to read real quotes from published authors. 